\title{
THE STEKLOV AND LAPLACIAN SPECTRA OF RIEMANNIAN MANIFOLDS WITH BOUNDARY
}

\author{
BRUNO COLBOIS, ALEXANDRE GIROUARD, AND ASMA HASSANNEZHAD
}

\begin{abstract}
Given two compact Riemannian manifolds $M_{1}$ and $M_{2}$ such that their respective boundaries $\Sigma_{1}$ and $\Sigma_{2}$ admit neighbourhoods $\Omega_{1}$ and $\Omega_{2}$ which are isometric, we prove the existence of a constant $C$ such that $\mid \sigma_{k}\left(M_{1}\right)-$ $\sigma_{k}\left(M_{2}\right) \mid \leq C$ for each $k \in \mathbb{N}$. The constant $C$ depends only on the geometry of $\Omega_{1} \cong \Omega_{2}$. This follows from a quantitative relationship between the Steklov eigenvalues $\sigma_{k}$ of a compact Riemannian manifold $M$ and the eigenvalues $\lambda_{k}$ of the Laplacian on its boundary. Our main result states that the difference $\left|\sigma_{k}-\sqrt{\lambda_{k}}\right|$ is bounded above by a constant which depends on the geometry of $M$ only in a neighbourhood of its boundary. The proofs are based on a Pohozaev identity and on comparison geometry for principal curvatures of parallel hypersurfaces. In several situations, the constant $C$ is given explicitly in terms of bounds on the geometry of $\Omega_{1} \cong \Omega_{2}$.
\end{abstract}

\section{INTRODUCTION}

Let $M$ be a smooth compact Riemannian manifold of dimension $n+1 \geq 2$, with nonempty boundary $\Sigma$. The Steklov eigenvalue problem on $M$ is to find all numbers $\sigma \in \mathbb{R}$ for which there exists a nonzero function $u \in C^{\infty}(M)$ which satisfies

$$
\begin{cases}\Delta u=0 & \text { in } M, \\ \frac{\partial u}{\partial \mathbf{n}}=\sigma u & \text { on } \Sigma .\end{cases}
$$

Here $\mathbf{n}$ is the outward unit normal along $\Sigma$ and $\Delta=\operatorname{div} \circ \nabla$ is the Laplace-Beltrami operator acting on $C^{\infty}(M)$. The Steklov problem has a discrete unbounded spectrum

$$
0=\sigma_{1} \leq \sigma_{2} \leq \sigma_{3} \leq \cdots \nearrow+\infty
$$

where each eigenvalue is repeated according to its multiplicity. For background on this problem, see [12, 20] and references therein.

1.1. The Dirichlet-to-Neumann map and spectral asymptotics. Let $\mathcal{H}$ : $C^{\infty}(\Sigma) \rightarrow C^{\infty}(M)$ be the harmonic extension operator: the function $u=\mathcal{H} f$ satisfies $u=f$ on $\Sigma$ and $\Delta u=0$ in $M$. The Steklov eigenvalues of $M$ are the eigenvalues of the Dirichlet-to-Neumann (DtN) map $D: C^{\infty}(\Sigma) \rightarrow C^{\infty}(\Sigma)$, which is defined by

$$
D f=\frac{\partial}{\partial \mathbf{n}} \mathcal{H} f
$$

1991 Mathematics Subject Classification. 35P15 (primary), 58C40, 35P20 (secondary).

Key words and phrases. Steklov eigenvalues, Pohozaev identity, comparison geometry, Riccati equation, Dirichlet-to-Neumann map. 
The DtN map is a first order self-adjoint elliptic pseudodifferential operator [27, pp. 37-38]. Its principal symbol is given by $p(x, \xi)=|\xi|$, while that of the Laplace operator $\Delta_{\Sigma}: C^{\infty}(\Sigma) \rightarrow C^{\infty}(\Sigma)$ is $|\xi|^{2}$. Standard elliptic theory [16, 26] then implies that

$$
\sigma_{k} \sim \sqrt{\lambda_{k}} \sim 2 \pi\left(\frac{k}{\omega_{n} \operatorname{Vol}_{n}(\Sigma)}\right)^{1 / n} \quad \text { as } \quad k \rightarrow \infty,
$$

where $\omega_{n}$ is the volume of the unit ball $B(0,1) \subset \mathbb{R}^{n}$. It follows that manifolds $M_{1}$ and $M_{2}$ which have isometric boundaries satisfy $\sigma_{k}\left(M_{1}\right) \sim \sigma_{k}\left(M_{2}\right)$ as $k \rightarrow \infty$.

It was proved in 21 that the full symbol of the DtN map is determined by the Taylor series of the Riemannian metric of $M$ in the normal direction along its boundary $\Sigma$ (see also [24]). Consider a closed Riemannian manifold $\left(\Sigma, g_{\Sigma}\right)$ and two compact Riemannian manifolds $\left(M_{1}, g_{1}\right)$ and $\left(M_{2}, g_{2}\right)$ with the same boundary $\Sigma=\partial M_{1}=\partial M_{2}$ such that $\left.g_{1}\right|_{\Sigma}=\left.g_{2}\right|_{\Sigma}=g_{\Sigma}$. If the metrics $g_{1}$ and $g_{2}$ have the same Taylor series on $\Sigma$, then $M_{1}$ and $M_{2}$ have asymptotically equivalent ${ }^{1}$ Steklov spectra:

$$
\sigma_{k}\left(M_{1}\right)=\sigma_{k}\left(M_{2}\right)+O\left(k^{-\infty}\right) \quad \text { as } \quad k \rightarrow \infty .
$$

See [11, Lemma 2.1]. In particular, $\lim _{k \rightarrow \infty} \sigma_{k}\left(M_{1}, g_{1}\right)-\sigma_{k}\left(M_{2}, g_{2}\right)=0$.

1.2. Main results. The asymptotic behavior described by (1) or (2) does not contain any information regarding an individual eigenvalue $\sigma_{k}$. In order to obtain such information, stronger hypothesis are needed. Indeed on any smooth compact Riemannian manifold $\left(M, g_{0}\right)$ with boundary, there exists a family of Riemannian metrics $\left(g_{\epsilon}\right)_{\epsilon \in \mathbb{R}_{+}}$such that $g_{\epsilon}=g_{0}$ on a neighbourhood $\Omega_{\epsilon}$ of $\Sigma=\partial M$, while for each $k \in \mathbb{N}$,

$$
\lim _{\epsilon \rightarrow \infty} \sigma_{k}\left(M, g_{\epsilon}\right)=0 .
$$

See [2] and [5] for two such constructions. If $n \geq 2$, there also exists a family of Riemannian metrics such that $g_{\epsilon}=g_{0}$ on a neighbourhood $\Omega_{\epsilon}$ of $\Sigma$ and

$$
\lim _{\epsilon \rightarrow \infty} \sigma_{2}\left(M, g_{\epsilon}\right)=+\infty \text {. }
$$

See 2] for the construction of these families. In this last construction, the neighbourhoods $\Omega_{\epsilon}$ are shrinking to $\Sigma$ as $\epsilon \rightarrow \infty$ :

$$
\bigcap_{\epsilon \in(0, \infty)} \Omega_{\epsilon}=\Sigma .
$$

However, if the manifolds $M_{1}$ and $M_{2}$ are uniformly isometric near their boundary, the situation is completely different.

Theorem 1. Given two compact Riemannian manifolds with boundary $M_{1}$ and $M_{2}$ such that their respective boundaries $\Sigma_{1}$ and $\Sigma_{2}$ admit neighbourhoods $\Omega_{1}$ and $\Omega_{2}$ which are isometric, there exists a constant $C$, which depends explicitly on the geometry of $\Omega_{1} \cong \Omega_{2}$, such that $\left|\sigma_{k}\left(M_{1}\right)-\sigma_{k}\left(M_{2}\right)\right| \leq C$ for each $k \in \mathbb{N}$.

\footnotetext{
${ }^{1}$ The notation $O\left(k^{-\infty}\right)$ designates a quantity which tends to zero faster than any power of $k$.
} 
Not only the difference $\left|\sigma_{k}\left(M_{1}\right)-\sigma_{k}\left(M_{2}\right)\right|$ is bounded for each given $k \in \mathbb{N}$, but the bound is uniform: the constant $C$ does not depend on $k$. The existence of $C$ could be obtained from a Dirichlet-to-Neumann bracketting argument, but this would not lead to an explicit expression.

Theorem 1 is a manifestation of the the principle stating that Steklov eigenvalues are mostly sensitive to the geometry of a manifold near its boundary. The more information we have on the metric $g$ on, and near, the boundary $\Sigma$, the more we can say about the Steklov spectrum of $M$. Knowing the metric on $\Sigma$ leads to the asymptotic formula (1), then knowing its Taylor series in the normal direction along $\Sigma$ leads to the refined asymptotic formula (2), and finally the knowledge of the metric on a neighbourhood of the boundary provides the extra information needed to control individual eigenvalues in Theorem 1

Remark 2. Let $\ell$ be the number of connected components of the boundary $\Sigma$. Under the hypothesis of Theorem 1 , it is well known that for $k \geq \ell$ the following holds:

$$
C^{-1} \leq \frac{\sigma_{k}\left(M_{1}\right)}{\sigma_{k}\left(M_{2}\right)} \leq C .
$$

This was used for instance in [2, 6]. Theorem 1 is stronger since it implies

$$
\frac{1}{1+\frac{C}{\sigma_{k}\left(M_{1}\right)}} \leq \frac{\sigma_{k}\left(M_{1}\right)}{\sigma_{k}\left(M_{2}\right)} \leq 1+\frac{C}{\sigma_{k}\left(M_{2}\right)} \text {. }
$$

It is also known that $\sigma_{k}\left(M_{i}\right) \geq \sigma_{k}^{N} \geq \sigma_{\ell}^{N}$, where $\sigma_{k}^{N}$ refer to the mixed NeumannSteklov problem on $\Omega_{1} \cong \Omega_{2}$. See [2] for details.

Theorem 1 follows from our main result, which is a quantitative comparison between the Steklov eigenvalues of $M$ and the eigenvalues of the Laplace operator $\Delta_{\Sigma}$ on its boundary $\Sigma$, which are denoted $0=\lambda_{1} \leq \lambda_{2} \leq \cdots \nearrow \infty$. Let $n \in \mathbb{N}$ and let $\alpha, \beta, \kappa_{-}, \kappa_{+} \in \mathbb{R}$ and $h>0$ be such that $\alpha \leq \beta$ and $\kappa_{-} \leq \kappa_{+}$. Consider the class $\mathcal{M}=\mathcal{M}\left(n, \alpha, \beta, \kappa_{-}, \kappa_{+}, h\right)$ of smooth compact Riemannian manifolds $M$ of dimension $n+1$ with nonempty boundary $\Sigma$, which satisfy the following hypotheses:

(H1) The rolling radius $2^{2}$ of $M$ satisfies $\bar{h}:=\operatorname{roll}(M) \geq h$.

(H2) The sectional curvature $K$ satisfies $\alpha \leq K \leq \beta$ on the tubular neighbourhood

$$
M_{\bar{h}}=\{x \in M: d(x, \Sigma)<\bar{h}\} .
$$

(H3) The principal curvatures of the boundary $\Sigma$ satisfy $\kappa_{-} \leq \kappa_{i} \leq \kappa_{+}$.

The main result of this paper is the following.

Theorem 3. There exist explicit constants $\mathbf{A}=\mathbf{A}\left(n, \alpha, \beta, \kappa_{-}, \kappa_{+}, h\right)$ and $\mathbf{B}=$ $\mathbf{B}\left(n, \alpha, \kappa_{-}, h\right)$ such that each manifold $M$ in the class $\mathcal{M}$ satisfies the following inequalities for each $k \in \mathbb{N}$,

$$
\begin{gathered}
\lambda_{k} \leq \sigma_{k}^{2}+\mathbf{A} \sigma_{k}, \\
\sigma_{k} \leq \mathbf{B}+\sqrt{\mathbf{B}^{2}+\lambda_{k}} .
\end{gathered}
$$

In particular, for each $k \in \mathbb{N},\left|\sigma_{k}-\sqrt{\lambda_{k}}\right|<\max \{\mathbf{A}, 2 \mathbf{B}\}$.

\footnotetext{
${ }^{2}$ The rolling radius will be defined in Section 2
} 
Under the hypotheses of Theorem 3 one can take

$$
\mathbf{A}=\frac{n}{\tilde{h}}+\sqrt{|\alpha|+\kappa_{-}^{2}} \quad \text { and } \quad \mathbf{B}=\frac{1}{2 \bar{h}}+\frac{n}{2} \sqrt{|\alpha|+\kappa_{-}^{2}},
$$

where $\tilde{h} \leq \bar{h}$ is a positive constant depending on $\bar{h}, \beta$, and $\kappa_{+}$. See $(10)$ for the precise definition. Various other values for the constants $\mathbf{A}$ and $\mathbf{B}$ will be given under more restrictive assumptions in Section 1.4

This theorem is in the spirit of the very nice result of [25] where a similar statement is proved for Euclidean domains. The dependence of $\mathbf{A}$ on the dimension $n$ is necessary. Indeed, it was observed in [25] that on the ball $B(0, R) \subset \mathbb{R}^{n+1}$ the following holds for each $k$ :

$$
\lambda_{k}=\sigma_{k}^{2}+\frac{n-1}{R} \sigma_{k} .
$$

We do not know if the dependence of $\mathbf{B}$ on $n$ is necessary in general. However, see Theorem 7 for situations where $\mathbf{B}$ does not depend on $n$.

Inequality (4) also holds under weaker hypotheses on $M$. Let $\mathcal{M}^{\prime}=\mathcal{M}^{\prime}\left(n, \alpha, \kappa_{-}, h\right)$ be the class of smooth compact Riemannian manifolds of dimension $n+1$ with nonempty boundary $\Sigma$, which satisfy (H1) and

$\left(\mathrm{H}^{\prime}\right)$ The Ricci curvature satisfies Ric $\geq n \alpha$ on $M_{\bar{h}}$.

$\left(\mathrm{H} 3^{\prime}\right)$ The mean curvature of $\Sigma$ satisfies $H \geq \kappa_{-}$.

Then we have the following theorem.

Theorem 4. There exists an explicit constant $\mathbf{B}=\mathbf{B}\left(n, \alpha, \kappa_{-}, h\right)$ such that each manifold $M$ in the class $\mathcal{M}^{\prime}$ satisfies the following inequality for each $k \in \mathbb{N}$,

$$
\sigma_{k} \leq \mathbf{B}+\sqrt{\mathbf{B}^{2}+\lambda_{k}} .
$$

1.3. Discussion and previous results. Quantitative estimates relating individual Steklov eigenvalues to eigenvalues of the tangential Laplacian $\Delta_{\Sigma}$ have been studied in $28,6,2,3,19,30,25,29$. They are relatively easy to obtain if the manifold $M$ is isometric (or quasi-isometric with some control) to a product near its boundary. See for example [3, Lemma 2.1]. In this context however, it is usually the quotient $\sqrt{\lambda_{k}} / \sigma_{k}$ which is controlled.

Theorem 3 is a generalization of the fundamental result of [25], and also of [29]. For bounded Euclidean domains $\Omega \subset \mathbb{R}^{n+1}$ with smooth connected boundary $\Sigma=$ $\partial \Omega$, Provenzano and Stubbe [25] proved a comparison result similar to Theorem 3 . with the constants $\mathbf{A}$ and $\mathbf{B}$ replaced by a constant $\mathbf{C}_{\Omega}$ depending on the dimension, the maximum of the mean of the absolute values of the principal curvatures on $\Sigma=\partial \Omega$ and the rolling radius of $\Omega$. Their main insight was to use a generalized Pohozaev identity ${ }^{3}$ in order to compare the Dirichlet energy of an harmonic function $u \in C^{\infty}(\bar{\Omega})$ to the $L^{2}$-norm of its normal derivative along $\Sigma$. In [29], Xiong extended the results of 25 to the Riemannian setting under rather stringent hypotheses. Indeed he considered domains $\Omega$ with convex boundary in a complete Riemannian manifold $X$ with either nonpositive $\left(K_{X} \leq 0\right)$ or strictly positive $\left(K_{X}>0\right)$ sectional

${ }^{3}$ See Section 3 for details on the Pohozaev identity. 
curvature, with some hypotheses on the shape operator (or second fundamental form). These condition in particular implies that the boundary is connected.

Theorem 3 improves these results in several ways. First we consider compact Riemannian manifolds with boundary. This is more general than bounded domains in a complete manifold (see 23 for a discussion of this question). Another strength of Theorem 3 is that we require geometric control of the manifold $M$ only in the neighbourhood $M_{\bar{h}}$ of its boundary. Finally, we are neither assuming the boundary $\Sigma$ to be connected nor the sectional curvature to have a constant sign.

Remark 5. Let $\ell$ be the number of connected components of $\Sigma$. Then $\lambda_{i}=0$, for $i=1, \ldots, \ell$. Thus, inequality (3) is of interest only for $k \geq \ell+1$. Furthermore inequality (4) becomes $\sigma_{k} \leq 2 \mathbf{B}$ for $k=1, \ldots, \ell$. There are indeed examples where $\sigma_{\ell}$ is arbitrarily small. See Section 6 .

Remark 6. The inner and exterior boundary of a domain $D \subset M$ are defined to be

$$
\partial_{I} D:=\partial D \cap \operatorname{int} M, \quad \partial_{E} D:=\bar{D} \cap \Sigma .
$$

The Cheeger and Jammes constant of $M$ are defined to be

$$
h_{M}=\inf _{D} \frac{\operatorname{Vol}_{n}\left(\partial_{I} D\right)}{\operatorname{Vol}_{n+1}(D)} \quad \text { and } \quad h_{M}^{\prime}=\inf _{D} \frac{\operatorname{Vol}_{n}\left(\partial_{I} D\right)}{\operatorname{Vol}_{n}\left(\partial_{E} D\right)},
$$

where both infima are taken over all domains $D \subset M$ such that $\operatorname{Vol}_{n+1}(D) \leq$ $\frac{\operatorname{Vol}_{n+1}(M)}{2}$. In [17] Jammes proved that

$$
\sigma_{2} \geq \frac{1}{4} h_{M} h_{M}^{\prime}
$$

Another useful lower bound can be deduced from Theorem 3 and the Cheeger inequality for $\Sigma$, which states that

$$
\lambda_{2} \geq \frac{1}{4} h_{\Sigma}^{2}
$$

where $h_{\Sigma}$ is the Cheeger constant of the closed manifold $\Sigma$. Indeed, it follows from inequality (3) and from inequality (7) that

$$
\sigma_{2} \geq \frac{1}{2}\left(-A+\sqrt{A^{2}+h_{\Sigma}^{2}}\right) .
$$

The interest of this lower bound is that it depends only on the geometry of $M$ on and near its boundary $\Sigma$. Of course this is useful only when the boundary $\Sigma$ is connected, in which case $h_{\Sigma}>0$. It is easy to construct examples of manifolds where $h_{M} h_{M}^{\prime}$ is arbitrarily small while $\frac{1}{2}\left(-A+\sqrt{A^{2}+h_{\Sigma}^{2}}\right)$ is bounded away from zero, for instance by attaching a Cheeger dumbbell with thin neck to the interior of $M$ using a thin cylinder.

One could also obtain similar lower bounds for $\sigma_{k}$ from the higher order Cheeger inequality for $\lambda_{k}$ [22, 10, which should be compared with the higher order Cheeger type inequality proved in [14. The lower bounds for $\sigma_{k}$ given in [17, 14] depends on the global geometry of the manifold and not only the geometry of the manifold near the boundary. 
1.4. Signed curvature and convexity. In 29], Xiong extended the results of [25] to domains $\Omega$ with convex boundary in a complete Riemannian manifold $X$ with either nonpositive $\left(K_{X} \leq 0\right)$ or strictly positive $\left(K_{X}>0\right)$ sectional curvature, with some hypotheses on the shape operator. This lead to explicit values for the constants $\mathbf{A}$ and $\mathbf{B}$. This work was enlightening for us, and lead to Theorem 7 and Corollary 8 below.

Let us now discuss various geometric settings where the constants $\mathbf{A}$ and $\mathbf{B}$ can be improved.

Theorem 7. Let $M$ be a smooth compact manifold of dimension $n+1$ with nonempty boundary $\Sigma$. Let $\bar{h}=\operatorname{roll}(M)$. Let $\lambda, \kappa_{+}>0$.

(1) Suppose that $\Sigma$ is totally geodesic. If $|K| \leq \lambda^{2}$, then (3) and (4) hold with

$$
\mathbf{A}=n \max \left\{\bar{h}^{-1}, \frac{2 \lambda}{\pi}\right\}+\lambda \quad \text { and } \quad \mathbf{B}=\frac{1}{2}\left(\frac{1}{\bar{h}}+n \lambda\right) .
$$

Knowing the sign of the sectional curvature $K$ leads to slightly more precise bounds.

a) If $-\lambda^{2}<K<0$, then (3) and (4) hold with

$$
\mathbf{A}=\frac{1}{\bar{h}}+\lambda \quad \text { and } \quad \mathbf{B}=\frac{1}{2}\left(\frac{1}{\bar{h}}+n \lambda\right) .
$$

b) $0<K<\lambda^{2}$, then (3) and (4) hold with

$$
\mathbf{A}=n \max \left\{\bar{h}^{-1}, \frac{2 \lambda}{\pi}\right\} \quad \text { and } \quad \mathbf{B}=\frac{1}{2 \bar{h}} .
$$

(2) Suppose that $\Sigma$ is a minimal hypersurface. If Ric $\geq \lambda^{2} n$, then (4) holds with $\mathbf{B}$ given in part (1).

(3) If $-\lambda^{2} \leq K \leq 0$ and each principal curvature satisfies $\lambda<\kappa_{i}<\kappa_{+}$, then (3) and (4) hold with

$$
\mathbf{A}=n \max \left\{\kappa_{+}, \frac{1}{\bar{h}}\right\} \quad \text { and } \quad \mathbf{B}=\frac{1}{2 \bar{h}} .
$$

(4) If $0<K<\lambda^{2}$ and each principal curvature satisfies $0<\kappa_{i}<\kappa_{+}$, then (3) and (4) hold with

$$
\mathbf{A}=n \max \left\{\frac{1}{\bar{h}}, \sqrt{\lambda^{2}+\kappa_{+}^{2}}\right\} \quad \text { and } \quad \mathbf{B}=\frac{1}{2 \bar{h}} .
$$

We recover the results in [29] as a consequence of Theorem 7 .

Corollary 8. [29, Theorem 1] Let $M$ be a domain in a complete Riemannian manifold with boundary $\Sigma$. Let $\lambda, \kappa_{+}>0$. Then

(1) If $-\lambda^{2} \leq K \leq 0$ and each principal curvature of $\Sigma$ satisfies $\lambda<\kappa_{i}<\kappa_{+}$, then (3) and (4) hold with

$$
\mathbf{A}=n \kappa_{+} \quad \text { and } \quad \mathbf{B}=\frac{1}{2} \kappa_{+} .
$$


(2) If $0<K<\lambda^{2}$ and each principal curvature of $\Sigma$ satisfies $0<\kappa_{i}<\kappa_{+}$, then (3) and (4) hold with

$$
\mathbf{A}=n \sqrt{\lambda^{2}+\kappa_{+}^{2}} \quad \text { and } \quad \mathbf{B}=\frac{1}{2} \sqrt{\lambda^{2}+\kappa_{+}^{2}} .
$$

We refer the reader to Corollary 35 for another situation where $\mathbf{A}$ and $\mathbf{B}$ have a simpler expression than those in Theorem 7

Let us conclude with the situation where $K \equiv 0$, which is motivated by the Euclidean case from [25].

Theorem 9. Let $M$ be a smooth compact manifold with nonempty boundary $\Sigma$. Suppose that $M$ is flat $(K \equiv 0)$.

- If $\kappa_{-} \leq \kappa \leq \kappa_{+}$, then (3) and (4) hold with

$$
\mathbf{A}=n \max \left\{\bar{h}^{-1}, \kappa_{+}\right\}+\left|\kappa_{-}\right| \quad \text { and } \quad \mathbf{B}=\frac{1}{2}\left(\bar{h}^{-1}+n\left|\kappa_{-}\right|\right) .
$$

- If $0 \leq \kappa_{i}<\kappa_{+}$, then (3) and (4) hold with

$$
\mathbf{A}=n \max \left\{\bar{h}^{-1}, \kappa_{+}\right\} \quad \text { and } \quad \mathbf{B}=\frac{1}{2 \bar{h}} .
$$

- If $\kappa_{-}<\kappa_{i} \leq 0$, then (3) and (4) hold with

$$
\mathbf{A}=\bar{h}^{-1}+\left|\kappa_{-}\right| \quad \text { and } \quad \mathbf{B}=\frac{1}{2}\left(\bar{h}^{-1}+n\left|\kappa_{-}\right|\right) .
$$

The proof of Theorem 9 is presented in Section 5.5

One could also consider manifolds whose boundary admits a neighbourhood which is isometric to the Riemannian product $[0, L) \times \Sigma$ for some $L>0$, in which case one can take $2 \mathbf{B}=\mathbf{A}=1 / L$. This is discussed in Section 5.6.

Plan of the paper. The proof of our main result (Theorem 3) is based on the comparison geometry of principal curvatures of hypersurfaces that are parallel to the boundary. This is presented in Section 2 following a review of relevant Jacobi fields and Riccati equations. The Pohozaev identity is used in Section 3 to relate the Dirichlet energy of an harmonic function to the $L^{2}$-norm of its normal derivative. The proof of Theorem 3 is presented in Section 4. In Section 5 we specialize to various geometrically rigid settings and prove Theorem 7. Here, we give precise values of the constants $\mathbf{A}, \mathbf{B}$ occurring in the estimates. In order to do this, we need some 1-dimensional calculations for specific Riccati equations, which are treated in an appendix at the end of the paper. In Section 6, we give various examples to illustrate the necessity of the geometric hypothesis occurring in Theorem 3 and Theorem 7 .

\section{Preliminaries from Riemannian geometry}

Let $M$ be a smooth compact Riemannian manifold of dimension $n+1$ with boundary $\Sigma$. The distance function $f: M \rightarrow \mathbb{R}$ to the boundary $\Sigma$ is given by

$$
f(x)=\operatorname{dist}(x, \Sigma) .
$$


Any $s \geq 0$ that is small enough is a regular value of $f$, so that the level sets $\Sigma_{s}:=f^{-1}(s)$ are submanifolds of $M$, which are called parallel hypersurfaces. They are the boundary of $\Omega_{s}:=\{x \in M: f(x) \geq s\}$. For $x \in \Sigma_{s}$, the gradient of the distance function $\nabla f(x)$, is the inward normal vector to $\Sigma_{s}=\partial \Omega_{s}$ :

$$
\boldsymbol{\nu}(x):=\nabla f(x) .
$$

In particular, for $x \in \Sigma, \boldsymbol{\nu}(x)=\nabla f(x)=-\mathbf{n}(x)$. The distance function $f$ satisfies $|\nabla f|=1$, whence the integral curves of $\nabla f$ are geodesics. That is,

$$
\nabla_{\nabla f} \nabla f=0 .
$$

2.1. Cut locus and rolling radius. Given $p \in \Sigma$, the exponential map defines a normal geodesic curve $\gamma_{p}: \mathbb{R}_{+} \longrightarrow M$,

$$
\gamma_{p}(s)=E_{s}(p)=\exp _{p}(s \boldsymbol{\nu}) .
$$

The cut point $\operatorname{cut}_{\Sigma}(p)$ of $p \in \Sigma$ is the point $E_{s_{0}}(p)$, where $s_{0}>0$ is the first time that $E_{s}(p)$ stops minimizing the distance to $\Sigma$. The cut locus of $\Sigma$ is

$$
\mathcal{C}_{\Sigma}=\left\{\operatorname{cut}_{\Sigma}(p): p \in \Sigma\right\} .
$$

The distance between $\Sigma$ and $\mathcal{C}_{\Sigma}$ is called the rolling radius ${ }^{4}$ of $M$ :

$$
\operatorname{roll}(M)=\operatorname{dist}\left(\Sigma, \mathcal{C}_{\Sigma}\right)
$$

Given $h \in(0, \operatorname{roll}(M)]$, define the tubular neighbourhood

$$
M_{h}=\{x \in M: f(x)<h\} .
$$

For each $x \in M_{h}$, there is exactly one nearest point to $x$ in $\Sigma$ and the exponential map $E_{s}(p)=\exp _{p}(s \boldsymbol{\nu}(p))$ defines a diffeomorphism between $[0, h) \times \Sigma$ and the tubular neighbourhood $M_{h}$. Moreover, for each $s \in[0, \operatorname{roll}(M))$, the map $E_{s}: \Sigma \rightarrow$ $\Sigma_{s}$ is a diffeomorphism.

2.2. The principal curvatures of parallel hypersurfaces. For each $s \in[0, \operatorname{roll}(M))$, the shape operator associated to the parallel hypersurface $\Sigma_{s}$ is the endomorphism $S_{s}: T \Sigma_{s} \rightarrow T \Sigma_{s}$ defined by

$$
S_{s}(X)=\nabla_{X} \nabla f
$$

For each tangent vectors $X, Y \in T \Sigma_{s}$, the following holds:

$$
\left\langle S_{s}(X), Y\right\rangle=\left\langle\nabla_{X} \nabla f, Y\right\rangle=\nabla^{2} f(X, Y)=\left\langle X, S_{s}(Y)\right\rangle,
$$

where $\langle X, Y\rangle$ is the Riemannian product and $\nabla^{2} f$ is the Hessian of $f$. In other words, the Hessian $\nabla^{2} f$ restricted to $\Sigma_{s}$ is its second fundamental form. The principal curvatures $\kappa_{i}(x), i=1, \ldots, n$, of $\Sigma_{s}$ at point $x$ with respect to outward normal vector $\mathbf{n}(x):=-\nabla f(x)$ are the eigenvalues of

$$
-S_{s}: T_{x} \Sigma_{s} \rightarrow T_{x} \Sigma_{s}
$$

In other words, the eigenvalues of the Hessian $\nabla^{2} f$ at $x \in \Sigma_{s}$, are

$$
0,-\kappa_{1}(x), \ldots,-\kappa_{n}(x) .
$$

The choice of sign is such that the principal curvatures of the boundary of Euclidean balls are positive.

\footnotetext{
${ }^{4}$ It is called the rolling radius because any open ball of radius $\leq \operatorname{roll}(M)$ can roll along $\Sigma$ while always remaining a subset of $M$.
} 
Our main geometric tools is a comparison estimate for the principal curvatures $\kappa_{i}$ of the parallel hypersurfaces $\Sigma_{s}$. This is adapted from the main theorem in [9] and from [8, Proposition 2.3].

Theorem 10 (Principal curvature comparison theorem I). Let $M$ be a smooth compact manifold with nonempty boundary $\Sigma$. Suppose the sectional curvature satisfies $\alpha \leq K$ and that the principal curvatures of the boundary satisfies $\kappa_{-} \leq \kappa_{i}$. Let $a:\left[0, m\left(\alpha, \kappa_{-}\right)\right) \rightarrow \mathbb{R}$ be the solution of

$$
a^{\prime}+a^{2}+\alpha=0, \quad a(0)=-\kappa_{-},
$$

with maximal existence time $m\left(\alpha, \kappa_{-}\right)$. Then $m\left(\alpha, \kappa_{-}\right) \geq \operatorname{roll}(M)$ and for each $\delta \in(0, \operatorname{roll}(M))$ the shape operator $S_{\delta}$ associated to the parallel hypersurface $\Sigma_{\delta}$ satisfies

$$
S_{\delta} \leq a(\delta) I
$$

That is, each principal curvature of $\Sigma_{\delta}$ satisfies $\kappa_{i}(\delta) \geq-a(\delta)$.

Remark 11. Theorem 10 gives an immediate upper bound for $\operatorname{roll}(M)$ :

$$
\operatorname{roll}(M) \leq m\left(\alpha, \kappa_{-}\right) .
$$

In Lemma 18 below, an explicit formula for $m\left(\alpha, \kappa_{-}\right)$is given. It could be compared with the result by Donnelly and Lee in [7. Theorem 3.1] on estimates for $\operatorname{roll}(M)$ on strictly convex domains, i.e. all eigenvalues of $-S$ are positive.

We also need a lower bound for the shape operators $S_{\delta}$ of parallel hypersurfaces in a neighbourhood of the boundary $\Sigma$. This requires further constraints on the neighbourhood.

Theorem 12 (Principal curvature comparison theorem II). Let $M$ be a smooth compact manifold with nonempty boundary $\Sigma$. Suppose the sectional curvature satisfies $K \leq \beta$ and that the principal curvatures of the boundary satisfies $\kappa_{i} \leq \kappa_{+}$. Let $\beta_{+}:=\max \{0, \beta\}$, and let $b:\left[0, m\left(\beta_{+}, \kappa_{+}\right)\right) \rightarrow \mathbb{R}$ be the solution of

$$
b^{\prime}+b^{2}+\beta_{+}=0, \quad b(0)=-\kappa_{+},
$$

with maximal existence time $m\left(\beta_{+}, \kappa_{+}\right)$. Let

$$
\tilde{h}:=\min \left\{m\left(\beta_{+}, \kappa_{+}\right), \operatorname{roll}(M)\right\} \text {. }
$$

Then for each $\delta \in(0, \tilde{h})$ the shape operator $S_{\delta}$ associated to the parallel hypersurface $\Sigma_{\delta}$ satisfies

$$
S_{\delta} \geq b(\delta) I
$$

That is, each principal curvatures of $\Sigma_{\delta}$ satisfies $\kappa_{i} \leq-b(\delta)$.

Remark 13. Theorem 12 also holds with $\beta_{+}$replaced by $\beta$, so that $\tilde{h}$ can be replaced by $\min \left\{m\left(\beta, \kappa_{+}\right), \operatorname{roll}(M)\right\}$. However, in our applications of Theorem 12, it is required that we use comparison with a space of non-negative sectional curvature. See Lemma 22 and Lemma 19.

Remark 14. Theorems 10 and 12 compare the evolution of the maximal and minimal principal curvatures of parallel hypersurfaces to those of umbilical hypersurfaces in model spaces of constant curvature $K \equiv \alpha, \beta_{+}$. Indeed, the functions $-a$ represent the principal curvatures of parallel umbilical hypersurfaces in a space form of constant curvature $K \equiv \alpha$, and similarly for the functions $-b$, for constant curvature $K \equiv \beta_{+}$. 
In our proofs, we will use mostly the following corollary in combination with explicit formulas for $a(s)$ and $b(s)$.

Corollary 15. Let $M$ be a smooth compact manifold with nonempty boundary $\Sigma$. Suppose the sectional curvature satisfies $\alpha \leq K \leq \beta$ and that each principal curvatures along the boundary satisfies $\kappa_{-} \leq \kappa_{i} \leq \kappa_{+}$. Then for each $\delta \in(0, \tilde{h})$ the principal curvatures of the parallel hypersurface $\Sigma_{\delta}$ satisfies

$$
-a(\delta) \leq \kappa_{i} \leq-b(\delta)
$$

Moreover, the left-hand side inequality holds for each $\delta \in(0, \bar{h})$.

Theorem 10 and Theorem 12 are sufficient to prove a version of Theorem 3 . Nevertheless, the following mean curvature comparison theorem leads to better bounds on the constant $\mathbf{B}$.

Theorem 16 (Mean curvature comparison theorem). Let $M$ be a smooth compact manifold with nonempty boundary $\Sigma$. Suppose the Ricci curvature satisfies Ric $\geq$ $n \alpha$ and that the mean curvature $H=-\frac{\operatorname{tr} S_{0}}{n}$ of the boundary satisfies $H \geq \kappa_{-}$. Let $\mu:\left[0, m\left(\alpha, \kappa_{-}\right)\right) \rightarrow \mathbb{R}$ be the solution of

$$
\mu^{\prime}+\mu^{2}+\alpha=0, \quad \mu(0)=-\kappa_{-},
$$

with maximal existence time $m\left(\alpha, \kappa_{-}\right)$. Then $m\left(\alpha, \kappa_{-}\right) \geq \bar{h}$ and for each $\delta \in(0, \bar{h})$ the mean curvature of the parallel hypersurface $\Sigma_{\delta}$ satisfies

$$
H(\delta)>-\mu(\delta) \text {. }
$$

2.3. Jacobi fields and Riccati equations. For the convenience of the reader, we explain briefly in this section how Theorem 10 and Theorem 12 are direct consequences of [8] and [9].

Given $v \in T_{p_{0}} \Sigma$, let $p:(-\epsilon, \epsilon) \rightarrow \Sigma$ be a smooth curve with $p(0)=p_{0}$ and $p^{\prime}(0)=v$. Then

$$
J(s):=\left.\frac{d}{d t} E_{s}(p(t))\right|_{t=0} \in T \Sigma_{s}
$$

defines a Jacobi field along the normal geodesic $\gamma(s):=E_{s}\left(p_{0}\right)$. This means that $J(s)$ satisfies the Jacobi equation

$$
J^{\prime \prime}(s)+R_{\nu}(J(s))=0,
$$

where $R_{\boldsymbol{\nu}}(J(s)):=R(J(s), \boldsymbol{\nu}) \boldsymbol{\nu}$ is the curvature tensor in the normal direction $\boldsymbol{\nu}=\nabla f$ to $\Sigma_{s}$. The field $J$ satisfies the initial conditions

$$
J(0)=v, \quad J^{\prime}(0)=S(v) .
$$

The shape operator is a tensor in $T \Sigma \otimes T^{\star} \Sigma$, which satisfies

$$
S_{s}(J(s))=J^{\prime}(s) \text {. }
$$

Its covariant derivative is given by

$$
\left(D_{X} S\right) V=D_{X}(S V)-S D_{X} V .
$$

Differentiating (13) and using (14) and the Jacobi equation (12) leads to the Riccati equation

$$
S_{s}^{\prime}(J(s))+S_{s}^{2}(J(s))+R_{\nu}(J(s))=0 .
$$


Given $p_{0} \in \Sigma$, consider the integral geodesic curve $\gamma:\left[0, \operatorname{cut}_{\Sigma}\left(p_{0}\right)\right] \longrightarrow M$ of $\nabla f$ starting at $\gamma(0)=p_{0}$. Identifying vectors in $T_{\gamma(s)} M$ with $T_{p_{0}} M$ via parallel transport along $\gamma$, we can consider $S_{s}$ and $R_{\nu}$ as endomorphisms on a single vector space $T_{p_{0}} \Sigma$. They satisfy the following matrix-valued Riccati equation in $\operatorname{End}\left(T_{p_{0}} \Sigma\right)$ :

$$
S^{\prime}(s)+S^{2}(s)+R(s)=0 .
$$

See [13, Chapter 3] for an enlightening discussion of Riccati equations for shape operators.

Let $E$ be a finite-dimensional real vector space with an euclidean inner product $\langle\cdot, \cdot\rangle$. Let $S(E)$ be the space of self-adjoint endomorphisms over $E$. For $A, B \in$ $S(E)$, we say $A \leq B$ if $B-A$ is positive semi definite. Then, we have

Theorem 17 (Riccati comparison theorem [9]). Let $R_{1}, R_{2}: \mathbb{R} \rightarrow S(E)$ be smooth curves with $R_{1} \geq R_{2}$. Let $S_{i}:\left[s_{0}, s_{i}\right) \rightarrow S(E)$ be a solution of

$$
S_{i}^{\prime}+S_{i}^{2}+R_{i}=0, \quad i=1,2
$$

with maximal existence time $s_{i} \in\left(s_{0}, \infty\right]$. Assume that $S_{1}\left(s_{0}\right) \leq S_{2}\left(s_{0}\right)$. Then $s_{1} \leq s_{2}$ and $S_{1}(s) \leq S_{2}(s)$ on $\left(s_{0}, s_{1}\right)$.

Proof of Theorem 10 and Theorem 12, Let $\mathbb{M}^{n+1}$ be a space form of sectional curvature $K$. Then $R=K I_{n}$ in (16), where $I_{n}$ is the identity matrix. Moreover, if we assume that $\mathbb{M}$ is simply connected and $\Sigma$ is an umbilical hypersurface i.e. $S(0)=-\kappa I_{n}$, then the Riccati equation (16) reduces to the one dimensional problem

$$
\left\{\begin{array}{l}
y^{\prime}+y^{2}+K=0 \\
y(0)=-\kappa
\end{array}\right.
$$

Its solutions describe the shape operator of a family of parallel umbilical hypersurfaces in $\mathbb{M}$. The idea of the proof is to compare the situation of the manifold $M$ with the situation of an umbilical hypersurface on a space form, where it is possible to do exact computations. Theorems 10 and 12 say that we can estimate the situation on $M$ by comparison with umbilical hypersurfaces on a space form, and in the sequel (Lemma 18) we will produce exact computations in these particular situations.

For the proof of Theorem 10, we apply Theorem 17 with $R_{1}=R$, where $R$ is given in (16), $R_{2}=\alpha I, S_{1}(0)=S(0)$ and $S_{2}(0)=-\kappa_{-} I$. By hypothesis, we have $R_{1} \geq R_{2}$ and $S_{1}(0) \leq S_{2}(0)$, so that we can apply Theorem 17. Moreover, $s_{1} \leq s_{2}$ implies that the principal curvature of the hypersurfaces level degenerate before $s_{2}$ so that the rolling radius has to be less than $s_{2}=m\left(\alpha, \kappa_{-}\right)$.

For the proof of Theorem 12, we apply Theorem 17 with $R_{1}=\beta_{+} I, R_{2}=R$, $S_{1}(0)=-\kappa_{+} I$ and $S_{2}(0)=S(0)$. By hypothesis, we have $R_{1} \geq R_{2}$ and $S_{1}(0) \leq$ $S_{2}(0)$, so that we again apply Theorem 17 . However the results of Theorem 17 are available only when the solution on $M$ is defined: we have to be less than $s_{1}=m\left(\beta_{+}, \kappa_{+}\right)$but also less than $\operatorname{roll}(M)$.

Proof of Theorem 16. Taking the trace of the Riccati equation (16) and using the Schwarz inequality for endomorphisms $n \operatorname{tr} S^{2} \geq(\operatorname{tr} S)^{2}$ leads to the following 
Riccati inequality:

$$
\frac{\operatorname{tr}(S(s))^{\prime}}{n}+\left(\frac{\operatorname{tr}(S(s))}{n}\right)^{2}+\frac{\operatorname{tr} R}{n} \leq 0 .
$$

This can be compared with the one dimensional Riccati problem

$$
\left\{\begin{array}{l}
\mu^{\prime}+\mu^{2}+\alpha=0 \\
\mu(0)=-\kappa_{-}
\end{array}\right.
$$

using [18, Corollary 1.6.2] to get the desired inequality. See [18, Pages 181-182] and [8] for details.

2.4. The Riccati equation for umbilic hypersurfaces in spaceforms. In order to apply Corollary 15 it will be useful to know the solutions of the onedimensional Riccati equation explicitly. The following Lemma is adapted from [8].

Lemma 18. Let $K, \kappa \in \mathbb{R}$. Consider the Riccati initial value problem (18):

$$
\left\{\begin{array}{l}
y^{\prime}+y^{2}+K=0 \\
y(0)=-\kappa
\end{array}\right.
$$

Let $\lambda=\sqrt{|K|} \geq 0$. The solutions of 180 , together with maximal existence time $m(K, \kappa)$, are as follows:

a) In Euclidean spaces $K=0$, the umbilical hypersurfaces are given by spheres and hyperplanes.

\begin{tabular}{lcl} 
Description & Solution $y(s)$ & $m(K, \kappa)$ \\
\hline Contracting spheres $\kappa>0$ & $\frac{1}{s-\frac{1}{\kappa}}$ & $1 / \kappa$ \\
Expanding spheres $\kappa<0$ & $\frac{1}{s-\frac{1}{\kappa}}$ & $\infty$ \\
Parallel hyperplanes $\kappa=0$ & 0 & $\infty$
\end{tabular}

b) In the sphere $\mathbb{S}_{K}$ of constant curvature $K=\lambda^{2}$ with $\lambda>0$, the umbilical hypersurfaces are geodesic spheres.

$$
y(s)=-\lambda \tan \left(\lambda s+\arctan \left(\frac{\kappa}{\lambda}\right)\right) \quad \text { with } m(K, \kappa)=\frac{1}{\lambda}\left(\frac{\pi}{2}-\arctan \left(\frac{\kappa}{\lambda}\right)\right)=\frac{1}{\lambda} \operatorname{arcot}\left(\frac{\kappa}{\lambda}\right)
$$

c) In the hyperbolic space $\mathbb{H}_{K}$ of constant curvature $K=-\lambda^{2}<0$ with $\lambda>0$, the umbilical hypersurfaces are the following.

\begin{tabular}{lll} 
Description & Solution $y(s)$ & Maximal existence $m(K, \kappa)$ \\
\hline Hyperbolic subspace $\kappa=0$ & $\lambda \tanh (\lambda s)$ & $\infty$ \\
Hypercycle $|\kappa|<\lambda$ & $\lambda \tanh \left(\lambda s-\operatorname{arctanh}\left(\frac{\kappa}{\lambda}\right)\right)$ & $\infty$ \\
Expanding spheres $\kappa<-\lambda$ & $\lambda \operatorname{coth}\left(\lambda s-\operatorname{arcoth}\left(\frac{\kappa}{\lambda}\right)\right)$ & $\infty$ \\
Contracting spheres $\kappa>\lambda$ & $\lambda \operatorname{coth}\left(\lambda s-\operatorname{arcoth}\left(\frac{\kappa}{\lambda}\right)\right)$ & $\frac{1}{\lambda} \operatorname{arcoth}\left(\frac{\kappa}{\lambda}\right)$ \\
Horospheres $|\kappa|=\lambda$ & $\kappa$ & $\infty$
\end{tabular}


The proof of Lemma 18 is presented in an appendix. Let us conclude this paragraph with a technical lemma which will allow the control near the cut locus, in the situation where the principal curvatures of the level hypersurfaces could degenerate.

Lemma 19. Let $K \in \mathbb{R}_{\geq 0}$ and $\kappa \in \mathbb{R}$. Let $y: I \subset \mathbb{R} \rightarrow \mathbb{R}$ be a solution of the Riccati equation

$$
y^{\prime}+y^{2}+K=0, \quad y(0)=-\kappa,
$$

with maximal interval $I$. Then $-(h-s) y(s) \leq 1$ for any $0 \leq s<h \in I$.

The proof of Lemma 19 will be presented in the appendix.

\section{PohozaeV identity AND ITS APPliCAtion}

Let $u \in C^{\infty}(M)$ be an harmonic function. The main goal of this section is to obtain a quantitative comparison inequality relating the norms $\left\|\nabla_{\Sigma} u\right\|_{L^{2}(\Sigma)}$ and $\left\|\frac{\partial u}{\partial \mathbf{n}}\right\|_{L^{2}(\Sigma)}$. Here $\nabla_{\Sigma} u$ denotes the tangential gradient of a function $u \in H^{1}(\Sigma)$ which is the gradient of $u$ on $\Sigma$. To achieve this goal, we need the generalized Pohozaev identity for harmonic functions on $M$.

Lemma 20 (Generalized Pohozaev identity). Let $F: M \rightarrow T M$ be a Lipschitz vector field. Let $u \in C^{\infty}(M)$ with $\Delta u=0$ in $M$. Then

$$
\begin{aligned}
0=\int_{\Sigma} \frac{\partial u}{\partial \mathbf{n}}\langle F, \nabla u\rangle d V_{\Sigma}- & \frac{1}{2} \int_{\Sigma}|\nabla u|^{2}\langle F, \mathbf{n}\rangle d V_{\Sigma} \\
& +\frac{1}{2} \int_{M}|\nabla u|^{2} \operatorname{div} F d V_{M}-\int_{M}\left\langle\nabla_{\nabla u} F, \nabla u\right\rangle d V_{M},
\end{aligned}
$$

where $\nabla F$ denotes the covariant derivative of $F, \mathbf{n}$ is the unit outward normal vector field along $\Sigma$, and $d V_{M}$ and $d V_{\Sigma}$ are Riemannian volume elements of $M$ and $\Sigma$ respectively.

The proof of Lemma 20 was provided first in 25, Lemma 3.1] for Euclidean domains. For simply-connected domains in a complete Riemannian manifold a proof was provided in [29, Lemma 9]. See also [15, Section 3], where a more general version of (21), so-called the Rellich identity is discussed. For the sake of completeness, we also include a proof here, which works for each compact manifold with boundary.

Proof of Lemma 20. Working in normal coordinates at a point $p \in M$, we can proceed exactly as in $\mathbb{R}^{n}$ since only the value of the metric and of its first order derivative at $p$ are involved in the following local computation. It follows from $\Delta u=0$ that

$$
\operatorname{div}(\langle F, \nabla u\rangle \nabla u)=\langle\nabla\langle F, \nabla u\rangle, \nabla u\rangle=\left\langle\nabla_{\nabla u} F, \nabla u\right\rangle+\nabla^{2} u(F, \nabla u) .
$$

Moreover,

$$
\operatorname{div}|\nabla u|^{2} F=2 \nabla^{2} u(F, \nabla u)+|\nabla u|^{2} \operatorname{div} F .
$$


Therefore

$$
\operatorname{div}\left(\langle F, \nabla u\rangle \nabla u-\frac{1}{2}|\nabla u|^{2} F\right)=\left\langle\nabla_{\nabla u} F, \nabla u\right\rangle-\frac{1}{2}|\nabla u|^{2} \operatorname{div} F .
$$

Integrating this identity on $M$ and using the divergence theorem completes the proof.

3.1. Analytic estimate using distance function. Recall that $f: M \rightarrow \mathbb{R}$ is defined by $f(x)=\operatorname{dist}(x, \Sigma)$. Given $h \in(0, \operatorname{roll}(M))$ let $\tilde{f}: M_{h} \rightarrow \mathbb{R}$ be the distance function to $\Sigma_{h}$. That is

$$
\tilde{f}(x):=h-f(x)=\operatorname{dist}\left(x, \Sigma_{h}\right) .
$$

Lemma 21. Let $u \in C^{\infty}(M)$ be such that $\Delta u=0$ and let $\eta=\frac{1}{2} \tilde{f}^{2}$. Then

$$
\int_{\Sigma}\left|\nabla_{\Sigma} u\right|^{2}-\left(\frac{\partial u}{\partial \mathbf{n}}\right)^{2} d V_{\Sigma}=\frac{1}{h} \int_{M_{h}}\left(\Delta \eta|\nabla u|^{2}-2 \nabla^{2} \eta(\nabla u, \nabla u)\right) d V_{M}
$$

Proof. Define the vector field $F: M \rightarrow T M$ by

$$
F(x):= \begin{cases}\nabla \eta(x) & \text { if } x \in M_{h}, \\ 0 & \text { if } x \in M \backslash M_{h} .\end{cases}
$$

Because $\nabla \eta=\tilde{f} \nabla \tilde{f}$ on $M_{h}$, the vector field $F$ is Lipschitz on $M$. Note that for $u \in C^{\infty}(M)$ and any $p \in \Sigma$,

$$
|\nabla u(p)|^{2}=\left|\nabla_{\Sigma} u(p)\right|^{2}+\left(\frac{\partial u}{\partial \mathbf{n}}(p)\right)^{2} .
$$

It follows from the Pohozaev identity (21) that

$$
\begin{aligned}
& 2 \int_{\Sigma} \frac{\partial u}{\partial \mathbf{n}}\langle\nabla \eta, \nabla u\rangle d V_{\Sigma}-\int_{\Sigma}\left|\nabla_{\Sigma} u\right|^{2}\langle\nabla \eta, \mathbf{n}\rangle d V_{\Sigma} \\
&-\int_{\Sigma}\left(\frac{\partial u}{\partial \mathbf{n}}\right)^{2}\langle\nabla \eta, \mathbf{n}\rangle d V_{\Sigma}+\int_{M}|\nabla u|^{2} \Delta \eta d V_{M} \\
&-2 \int_{M}\langle\nabla \nabla u \nabla \eta, \nabla u\rangle d V_{M}=0 .
\end{aligned}
$$

Since $\left.\nabla \eta\right|_{\Sigma}=h \mathbf{n}$, we get

$$
\begin{aligned}
2 h \int_{\Sigma}\left(\frac{\partial u}{\partial \mathbf{n}}\right)^{2} d V_{\Sigma} & -h \int_{\Sigma}\left|\nabla_{\Sigma} u\right|^{2} d V_{\Sigma}-h \int_{\Sigma}\left(\frac{\partial u}{\partial \mathbf{n}}\right)^{2} d V_{\Sigma} \\
& +\int_{M}|\nabla u|^{2} \Delta \eta d V_{M}-2 \int_{M}\left\langle\nabla_{\nabla u} \nabla \eta, \nabla u\right\rangle d V_{M}=0
\end{aligned}
$$


3.2. Geometric control on a tubular neighbourhood. Let $M$ be a smooth compact Riemannian manifold with nonempty boundary $\Sigma$ and rolling radius $\bar{h}=$ $\operatorname{roll}(M)$. Suppose the sectional curvature satisfies $\alpha \leq K \leq \beta$ on $M_{\bar{h}}$ and suppose the principal curvatures of $\Sigma$ satisfy $\kappa_{-} \leq \kappa_{i} \leq \kappa_{+}$.

The link between the function $\eta=\frac{1}{2} \tilde{f}^{2}$ and the local geometry of parallel hypersurfaces is established in the following lemma.

Lemma 22. Let $h \in(0, \tilde{h})$ and let $\delta \in(0, h)$. Then the eigenvalues of the Hessian $\nabla^{2} \eta$ at $y \in \Sigma_{\delta}$ are

$$
\rho_{1}=(h-\delta) \kappa_{1}(y) \leq \ldots \leq \rho_{n}=(h-\delta) \kappa_{n}(y) \leq \rho_{n+1}=1 .
$$

Proof. The Hessian of $\eta$ is

$$
\nabla^{2} \eta=\nabla \tilde{f} \otimes \nabla \tilde{f}+\tilde{f} \nabla^{2} \tilde{f} .
$$

The eigenvalues of $\nabla^{2} \tilde{f}(y)$ are $0, \kappa_{1}(y), \ldots, \kappa_{n}(y)$. Moreover, for each $V, W \in T_{y} \Sigma_{\delta}$,

$$
\nabla \tilde{f} \otimes \nabla \tilde{f}(V, W)=0 .
$$

It follows from

$$
\Sigma_{\delta}=\tilde{f}^{-1}(h-\delta)
$$

that for each $i=1, \cdots, n$ the number $\rho_{i}:=(h-\delta) \kappa_{i}(y)$ is an eigenvalue of $\nabla^{2} f(y)$. Finally, it follows from Corollary 15 that $(h-\delta) \kappa_{i}(y) \leq-(h-\delta) b(\delta)$, where $b(\delta)$ is defined in (9) using $\beta_{+} \geq 0$. The proof is now completed using Lemma 19.

Lemma 23. For each smooth function $v \in C^{\infty}(M)$, the following pointwise estimate holds on $M_{\bar{h}}$,

$$
-\left(1-\sum_{i=1}^{n} \rho_{i}\right)|\nabla v|^{2} \leq \Delta \eta|\nabla v|^{2}-2 \nabla^{2} \eta(\nabla v, \nabla v) \leq\left(1+\sum_{i=2}^{n} \rho_{i}-\rho_{1}\right)|\nabla v|^{2} .
$$

Proof. Let $A=\operatorname{diag}\left(\rho_{i}\right)$ be the diagonal matrix representing the Hessian of $\eta$ at $x$ in an orthonormal frame. Let $w=\nabla v(x)$. Observe that

$$
\rho_{1}|w|^{2} \leq A w \cdot w \leq \rho_{n+1}|w|^{2}
$$

It follows that

$$
\left(\rho_{1}+\cdots+\rho_{n}-\rho_{n+1}\right)|w|^{2} \leq|w|^{2} \operatorname{tr}(A)-2 A w \cdot w \leq\left(-\rho_{1}+\rho_{2}+\cdots+\rho_{n+1}\right)|w|^{2},
$$

with $\rho_{n+1}=1$.

Lemma 24. Let $M \in \mathcal{M}\left(n, \alpha, \beta, \kappa_{+}, \kappa_{-}, \bar{h}\right)$. Then there exist constants $\overline{\mathbf{A}}=$ $\overline{\mathbf{A}}\left(n, \alpha, \beta, \kappa_{-}, \kappa_{+}\right)$and $\overline{\mathbf{B}}=\overline{\mathbf{B}}\left(n, \alpha, \kappa_{-}\right)$such that every $v \in H^{1}(M)$ satisfies the following inequality for each $h \in(0, \tilde{h})$,

$$
\begin{aligned}
-(1+\overline{\mathbf{B}}) \int_{M}|\nabla v|^{2} d V_{M} \leq \int_{M_{h}}|\nabla v|^{2} \Delta \eta-2 \nabla^{2} \eta & (\nabla v, \nabla v) d V_{M} \\
& \leq(1+\overline{\mathbf{A}}) \int_{M}|\nabla v|^{2} d V_{M} .
\end{aligned}
$$

In addition, the left-hand side inequality holds for each $h \in(0, \bar{h})$. 
Proof. Let $h \in(0, \tilde{h})$. Given $\delta \in(0, h)$, let $H(y)$ be the mean curvature of the parallel hypersurface $\Sigma_{\delta}$ at $y \in \Sigma_{\delta}$. It follows from Lemma 22 and Lemma 23 that

$$
\begin{aligned}
-(1-(h-\delta) n H)|\nabla v|^{2} \leq \Delta \eta|\nabla v|^{2}-2 \nabla^{2} \eta(\nabla v, \nabla v) & \\
& \leq\left(1+(h-\delta) \sum_{i=2}^{n} \kappa_{i}-(h-\delta) \kappa_{1}\right)|\nabla v|^{2} .
\end{aligned}
$$

Following Corollary 15 and Theorem 16, let

$$
\overline{\mathbf{A}}(h)=\max _{0 \leq \delta<h}\{(h-\delta)(a(\delta)-(n-1) b(\delta))\},
$$

and

$$
\overline{\mathbf{B}}(h)=n \max _{0 \leq \delta<h}(h-\delta) \mu(\delta),
$$

so that for each $h \in(0, \tilde{h})$ one has

$$
\begin{aligned}
-(1+\overline{\mathbf{B}}(h)) \int_{M}|\nabla v|^{2} d V_{M} \leq \int_{M_{h}}|\nabla v|^{2} \Delta \eta-2 \nabla^{2} \eta(\nabla v, \nabla v) d V_{M} & \\
& \leq(1+\overline{\mathbf{A}}(h)) \int_{M}|\nabla v|^{2} d V_{M} .
\end{aligned}
$$

Notice that according to Theorem 16, the left-hand side inequality holds for each $h \in(0, \bar{h})$. One can therefore take

$$
\overline{\mathbf{A}} \geq \max _{0 \leq h<\tilde{h}} \overline{\mathbf{A}}(h)
$$

and

$$
\overline{\mathbf{B}} \geq \max _{0 \leq h<\bar{h}} \overline{\mathbf{B}}(h) .
$$

It remains to prove that the right-hand sides of $(29)$ and (30) are finite. To achieve this, we provide upper bounds for $\bar{A}(h)$ and $\bar{B}(h)$.

For each $\delta \in(0, h)$, the number $-a(\delta)$ is the principal curvature of an umbilical hypersurface of principal curvature $\kappa_{-}$in the space form of constant curvature $\alpha$. Observe that for each $\epsilon>0$,

$$
\alpha \geq-(\overbrace{|\alpha|+\kappa_{-}^{2}+\epsilon}^{\hat{\alpha}}) .
$$

Using Corollary 15 one more time we see that $a(\delta)$ is bounded above by the solution $y(\delta)$ of

$$
y^{\prime}+y^{2}-\hat{\alpha}=0, \quad y(0)=-\kappa_{-} .
$$

Because $\left|\kappa_{-}\right| \leq \sqrt{\hat{\alpha}}$, Lemma 18 indicates that $y(\delta)$ is the principal curvature of an hypercycle in $\mathbb{H}_{\sqrt{\hat{\alpha}}}$ and it follows that for each $\delta \in(0, h)$,

$$
a(\delta) \leq \sqrt{\hat{\alpha}} \tanh (\star) \leq \sqrt{|\alpha|+\kappa_{-}^{2}} .
$$

Here $\star$ stands for the argument but is not relevant to the estimate. This bound does not depend on $0<h<\bar{h}$. We proceed similarly to bound $-(h-\delta) b(\delta)$ for each $h \in(0, \tilde{h})$. Indeed, the situation is simpler since we have defined $b(\delta)$ using 
a space of constant curvature $\beta_{+} \geq 0$. In this case it follows from Lemma 19 that $-(h-\delta) b(\delta) \leq 1$. Substitution in the definition (27) leads to

$$
\overline{\mathbf{A}}(h) \leq(\tilde{h}-h) \sqrt{|\alpha|+\kappa_{-}^{2}}+(n-1)
$$

This implies that 250 holds for any

$$
\overline{\mathbf{A}} \geq \tilde{h} \sqrt{|\alpha|+\kappa_{-}^{2}}+(n-1) .
$$

One proceed exactly in the same way to bound $(h-\delta) n \mu(\delta)$ and obtain

$$
(h-\delta) n \mu(\delta) \leq(\bar{h}-h) \sqrt{|\alpha|+\kappa_{-}^{2}} .
$$

This implies that (25) holds for any

$$
\overline{\mathbf{B}} \geq \bar{h} n \sqrt{|\alpha|+\kappa_{-}^{2}} .
$$

This completes the proof.

Note that to bound $\bar{B}(h)$ from above we use Theorem 16 , where only lower bounds on the Ricci and on the mean curvature are needed. Hence, we can weaken the hypotheses in Lemma 24 .

Lemma 25. Let $M \in \mathcal{M}^{\prime}\left(n, \alpha, \kappa_{-}, \bar{h}\right)$. Then there exists constant $\overline{\mathbf{B}}=\overline{\mathbf{B}}\left(n, \alpha, \kappa_{-}\right)$ such that every $v \in H^{1}(M)$ satisfies the following inequality for each $h \in(0, \bar{h})$,

$$
-(1+\overline{\mathbf{B}}) \int_{M}|\nabla v|^{2} d V_{M} \leq \int_{M_{h}}|\nabla v|^{2} \Delta \eta-2 \nabla^{2} \eta(\nabla v, \nabla v) d V_{M} .
$$

Remark 26. When the lower bound for the mean curvature of the parallel hypersurfaces is nonnegative or equivalently when $\mu(\delta)$ is nonpositive for every $0 \leq \delta \leq \bar{h}$, then we can simply take $\overline{\mathbf{B}}=0$.

Theorem 27. The assumptions are the same as in Lemma 24.

Let $\mathbf{A}, \mathbf{B} \in \mathbb{R}$ be such that

$$
\mathbf{A} \geq \frac{1}{\tilde{h}}(1+\overline{\mathbf{A}}), \quad \text { and } \quad \mathbf{B} \geq \frac{1}{2 \bar{h}}(1+\overline{\mathbf{B}}) .
$$

Let $v \in C^{\infty}(M)$ be such that $\Delta v=0$ in $M$ and normalized such that $\int_{\Sigma} v^{2} d V_{\Sigma}=1$. Then the following holds

$$
\begin{gathered}
\int_{\Sigma}\left|\nabla_{\Sigma} v\right|^{2} d V_{\Sigma} \leq \int_{\Sigma}\left(\frac{\partial v}{\partial \mathbf{n}}\right)^{2} d V_{\Sigma}+\mathbf{A}\left(\int_{\Sigma}\left(\frac{\partial v}{\partial \mathbf{n}}\right)^{2} d V_{\Sigma}\right)^{\frac{1}{2}} \\
\left(\int_{\Sigma}\left(\frac{\partial v}{\partial \mathbf{n}}\right)^{2} d V_{\Sigma}\right)^{\frac{1}{2}} \leq \mathbf{B}+\sqrt{\mathbf{B}+\int_{\Sigma}\left|\nabla_{\Sigma} v\right|^{2} d V_{\Sigma}}
\end{gathered}
$$


Proof. This is identical to the proof of Theorem 3.18 in [25], and included for the sake of completeness.

Proof of (34). It follows from Lemma 21 and Lemma 24 that

$$
\begin{aligned}
\int_{\Sigma}\left|\nabla_{\Sigma} v\right|^{2}-\left(\frac{\partial v}{\partial \mathbf{n}}\right)^{2} d V_{\Sigma} & =\frac{1}{h} \int_{M_{h}}|\nabla v|^{2} \Delta \eta-2 \nabla^{2} \eta(\nabla v, \nabla v) d V_{M} \\
& \leq \mathbf{A} \int_{M}|\nabla v|^{2} d V_{M}=\mathbf{A} \int_{\Sigma} v \frac{\partial v}{\partial \mathbf{n}} d V_{\Sigma} .
\end{aligned}
$$

It follows from the Cauchy-Schwarz inequality that

$$
\int_{\Sigma}\left|\nabla_{\Sigma} v\right|^{2}-\left(\frac{\partial v}{\partial \mathbf{n}}\right)^{2} d V_{\Sigma} \leq \mathbf{A}\left(\int_{\Sigma}\left(\frac{\partial v}{\partial \mathbf{n}}\right)^{2} d V_{\Sigma}\right)^{1 / 2}
$$

This completes the proof of 34 .

Proof of (35). We repeat the calculation above using Lemma 21 and Lemma 24

$$
\begin{aligned}
\int_{\Sigma}\left|\nabla_{\Sigma} v\right|^{2}-\left(\frac{\partial v}{\partial \mathbf{n}}\right)^{2} d V_{\Sigma} & =\frac{1}{h} \int_{M_{h}}|\nabla v|^{2} \Delta \eta-2 \nabla^{2} \eta(\nabla v, \nabla v) d V_{M} \\
& \geq-2 \mathbf{B} \int_{M}|\nabla v|^{2} d V_{M}=-2 \mathbf{B} \int_{\Sigma} v \frac{\partial v}{\partial \mathbf{n}} d V_{\Sigma} .
\end{aligned}
$$

It follows from the Cauchy-Schwarz inequality that

$$
\int_{\Sigma}\left|\nabla_{\Sigma} v\right|^{2}-\left(\frac{\partial v}{\partial \mathbf{n}}\right)^{2} d V_{\Sigma} \geq-2 \mathbf{B}\left(\int_{\Sigma}\left(\frac{\partial v}{\partial \mathbf{n}}\right)^{2} d V_{\Sigma}\right)^{1 / 2}
$$

We solve this inequality in terms of the unknown $\left(\int_{\Sigma}\left(\frac{\partial v}{\partial \mathbf{n}}\right)^{2} d V_{\Sigma}\right)^{1 / 2}$ and get

$$
\left(\int_{\Sigma}\left(\frac{\partial v}{\partial \mathbf{n}}\right)^{2} d V_{\Sigma}\right)^{1 / 2} \leq \mathbf{B}+\sqrt{\mathbf{B}^{2}+\int_{\Sigma}\left|\nabla_{\Sigma} v\right|^{2} d V_{\Sigma}}
$$

Remark 28. Note that inequality (35) is also true under the assumption of Lemma25.

Remark 29. It follows from the above that for each $f \in C^{\infty}(\Sigma)$ and $u=\mathcal{H} f \in$ $C^{\infty}(M)$,

$$
\left|\left\|\nabla_{\Sigma} f\right\|^{2}-\|D f\|^{2}\right| \leq \max \{A, 2 B\} \int_{M_{h}}|\nabla u|^{2} d V_{M}
$$

The tangential gradient and the DtN map have comparable norm, the difference being controlled by the Dirichlet energy of the harmonic extension.

Remark 30. If we keep the norm $\|v\|_{\Sigma}$ in the above proof of (35) we get from the Cauchy-Schwarz inequality that

$$
\int_{\Sigma}\left|\nabla_{\Sigma} v\right|^{2}-\left(\frac{\partial v}{\partial \mathbf{n}}\right)^{2} d V_{\Sigma} \geq-2 \mathbf{B}\left(\int_{\Sigma} v^{2} d V_{\Sigma}\right)^{1 / 2}\left(\int_{\Sigma}\left(\frac{\partial v}{\partial \mathbf{n}}\right)^{2} d V_{\Sigma}\right)^{1 / 2}
$$


And solving the inequality leads to

$$
\|D v\|_{L^{2}(\Sigma)} \leq \mathbf{B}\|v\|_{L^{2}(\Sigma)}+\sqrt{\mathbf{B}^{2}\|v\|_{L^{2}(\Sigma)}^{2}+\left\|\nabla_{\Sigma} v\right\|^{2}} \leq\left(\sqrt{\mathbf{B}^{2}+1}\right)\|v\|_{H^{1}(\Sigma)} .
$$

This proves that the DtN map $D: H^{1}(\Sigma) \rightarrow L^{2}(\Sigma)$ is a bounded map and that its operator norm is smaller than $\sqrt{\mathbf{B}^{2}+1}$. It is well-known that $D$ is bounded in this setting, but our result provide an explicit bound on its norm.

\section{Proof of the main Result}

We are now ready for the proof of the main result. The proof follows the same lines of argument as in [25, Theorem 1.7]. For the sake of completeness we give the proof here.

Proof of Theorem 3. Let us first recall the variational characterizations of the eigenvalues of Dirichlet-to-Neumann operator $\sigma_{k}$ and of the Laplacian $\lambda_{k}$. For each $k \in \mathbb{N}$,

$$
\begin{aligned}
& \sigma_{k}=\inf _{\substack{V \subset H^{1}(M), \operatorname{dim} V=k}} \sup _{\substack{v \in V, \int_{\Sigma} v^{2} d V_{\Sigma}=1}} \int_{M}|\nabla v|^{2} d V_{M} ; \\
& \lambda_{k}=\left.\inf _{\substack{V \subset H^{1}(\Sigma), \operatorname{dim} V=k}} \sup _{\substack{v \in V, \int_{\Sigma} v^{2} d V_{\Sigma}=1}} \int_{\Sigma}|\nabla|_{\Sigma} v\right|^{2} d V_{\Sigma} .
\end{aligned}
$$

Part a. Let $\left(\psi_{i}\right)_{i \in \mathbb{N}} \subset H^{1}(M)$ be a complete set of eigenfunctions corresponding to the Steklov eigenvalues $\sigma_{i}(M)$ such that their restrictions to $\Sigma$ form an orthonormal basis of $L^{2}(\Sigma)$. We use the space spanned by $\left\{\left.\psi_{i}\right|_{\Sigma}\right\}_{i=1}^{k}$ as a trial space $V$ in (37). Every $v \in V$ with $\int_{\Sigma} v^{2} d V_{\Sigma}=1$ can be written as $v=\left.\sum c_{i} \psi_{i}\right|_{\Sigma}$ with $\sum c_{i}^{2}=1$. Note that

$$
\frac{\partial \psi_{i}}{\partial \mathbf{n}}=\sigma_{i} \psi_{i}, \quad \text { on } \Sigma \quad \text { for } \quad i=1, \ldots, k .
$$

Hence, using inequality (34) we get

$$
\begin{aligned}
\lambda_{k} & \leq \sup _{\substack{0 \neq u \in V \\
0 \neq \\
\int_{\Sigma} u^{2} d V_{\Sigma}=1}} \int_{\Sigma}\left|\nabla_{\Sigma} v\right|^{2} d V_{\Sigma} \\
& \leq \sup _{\substack{0 \neq u \in V \\
\int_{\Sigma} u^{2} d V_{\Sigma}=1}} \int_{\Sigma}\left(\frac{\partial v}{\partial \mathbf{n}}\right)^{2} d V_{\Sigma}+\mathbf{A}\left(\int_{\Sigma}\left(\frac{\partial v}{\partial \mathbf{n}}\right)^{2} d V_{\Sigma}\right)^{\frac{1}{2}} \\
& =\sup _{\left(c_{1}, \cdots, c_{k}\right) \in \mathbb{S}^{k-1}}\left(\sum_{i=1}^{k} c_{i}^{2} \sigma_{i}^{2}+\mathbf{A}\left(\sum_{i=1}^{k} c_{i}^{2} \sigma_{i}^{2}\right)^{\frac{1}{2}}\right)=\sigma_{k}^{2}+\mathbf{A} \sigma_{k} .
\end{aligned}
$$

This proves inequality (3).

Part b. We prove inequality (4) in an analogous way. Let $\left\{\varphi_{i}\right\}_{i \in \mathbb{N}} \subset C^{\infty}(\Sigma)$ be an orthonormal basis of $L^{2}(\Sigma)$ such that $-\Delta_{\Sigma} \varphi_{i}=\lambda_{i} \varphi_{i}$ on $\Sigma$. 
Consider the harmonic extensions $u_{i}=\mathcal{H} \varphi_{i}$ for $i=1, \ldots, k$. They satisfy the following problem:

$$
\begin{cases}\Delta u_{i}=0, & \text { in } M \\ u_{i}=\varphi_{i}, & \text { on } \Sigma\end{cases}
$$

Let $V \subset H^{1}(M)$ be the space spanned by $u_{1}, \ldots u_{k}$. Any function $\phi \in V$ with $\int_{\Sigma} \phi^{2} d V_{\Sigma}=1$ can be written as $\phi=\sum_{i=1}^{k} c_{i} u_{i}$ with $\sum c_{i}^{2}=1$. Moreover $\Delta \phi=0$ for all $\phi \in V$. Using the min-max principle and inequality (35) together with the Cauchy-Schwarz inequality leads to

$$
\begin{aligned}
\sigma_{k} & \leq \sup _{\substack{0 \neq \phi \in V \\
\int_{\Sigma} \phi^{2} d V_{\Sigma}=1}} \int_{M}|\nabla \phi|^{2} d V_{M}=\sup _{\left(c_{1}, \ldots, c_{k}\right) \in \mathbb{S}^{k-1}} \int_{M}|\nabla \phi|^{2} d V_{M} \\
& \leq \max _{\left(c_{1}, \ldots, c_{k}\right) \in \mathbb{S}^{k-1}}\left(\int_{\Sigma}\left(\frac{\partial \phi}{\partial \mathbf{n}}\right)^{2} d V_{\Sigma}\right)^{\frac{1}{2}} \\
& \leq \mathbf{B}+\left(\mathbf{B}^{2}+\sup _{\left(c_{1}, \ldots, c_{k}\right) \in \mathbb{S}^{k-1}} \int_{\Sigma}\left|\nabla_{\Sigma} \phi\right|^{2}\right)^{\frac{1}{2}} \\
& \leq \mathbf{B}+\left(\mathbf{B}^{2}+\sup _{\left(c_{1}, \ldots, c_{k}\right) \in \mathbb{S}^{k-1}} \sum_{i=1}^{k} c_{i}^{2} \lambda_{i}\right)^{\frac{1}{2}}=\mathbf{B}+\sqrt{\mathbf{B}^{2}+\lambda_{k}} .
\end{aligned}
$$

This proves inequality (4).

Part c. It remains to prove that $\left|\sigma_{k}-\sqrt{\lambda_{k}}\right| \leq \max \{\mathbf{A}, 2 \mathbf{B}\}$. Indeed,

$$
\begin{aligned}
\sigma_{k}-\sqrt{\lambda_{k}} & \leq \mathbf{B}+\sqrt{\mathbf{B}^{2}+\lambda_{k}}-\sqrt{\lambda_{k}} \\
& =\mathbf{B}+\frac{\mathbf{B}^{2}}{\sqrt{\mathbf{B}^{2}+\lambda_{k}}+\sqrt{\lambda_{k}}} \leq 2 \mathbf{B} .
\end{aligned}
$$

Similarly,

$$
\sqrt{\lambda_{k}}-\sigma_{k}=\frac{\lambda_{k}-\sigma_{k}^{2}}{\sqrt{\lambda_{k}}+\sigma_{k}} \leq \frac{\mathbf{A} \sigma_{k}}{\sqrt{\lambda_{k}}+\sigma_{k}} \leq \mathbf{A}
$$

This implies $\left|\sigma_{k}-\sqrt{\lambda_{k}}\right| \leq \max \{\mathbf{A}, 2 \mathbf{B}\}$.

The proof of Theorem 1 is now a direct consequence.

Proof of Theorem 1 . Let $\Omega$ be a neighbourhood of the boundary $\Sigma$ and let $g_{1}, g_{2}$ be two Riemannian metrics which coincide on $\Omega$. Let $M_{\tilde{h}}$ be the admissible neighbourhood of $\Sigma$ for the metric $g_{1}$. Let $\hat{h} \in(0, \tilde{h})$ be the largest number such that $M_{\hat{h}} \subset \Omega$. One can then define $C>0$ using formula (33), for the metric $g_{1}$, with $\tilde{h}$ replaced by $\hat{h}$. Substituting $C$ for $\max \{\mathbf{A}, 2 \mathbf{B}\}$ in the proof of Theorem 3 leads to $\left|\sigma_{k}\left(g_{i}\right)-\sqrt{\lambda_{k}}\right|<C$ for $i=1,2$. This implies that $\left|\sigma_{k}\left(g_{1}\right)-\sigma_{k}\left(g_{2}\right)\right| \leq 2 C$.

Proof of Theorem 4. The proof is identical to that of Part $\mathbf{b}$ above, but we only need to use inequality (35) under the assumption of Lemma25 (see Remark 28). 


\section{Signed CURVATURE AND CONVEXity}

In this section we will give precise bounds on the constants $\mathbf{A}$ and $\mathbf{B}$ defined in (33) and prove Theorem 7. In the situation where the sectional curvature is constrained to have a constant sign and where we impose convexity assumptions on the boundary $\Sigma$, we recover and improve the results of Xiong [29].

Our strategy is to estimate the quantities $(\tilde{h}-\delta) a(\delta),(\tilde{h}-\delta) b(\delta)$ and $(\bar{h}-\delta) \mu(\delta)$ which appear in the definitions 29 and $(30)$ of the constants $\overline{\mathbf{A}}$ and $\overline{\mathbf{B}}$, and thus also in the definitions 33 of $\mathbf{A}$ and $\mathbf{B}$. This is achieved by using the explicit formulas for $a(\delta), b(\delta)$ and $\mu(\delta)$ provided by Lemma 18 together with some technical lemmas regarding solutions of the Riccati equation in dimension 1 . These are stated in the next paragraph.

5.1. Technical lemmas regarding Riccati equation in dimension 1 . In this paragraph we state a technical lemma which will be proved in the appendix. Its goal is to estimate the function $f(x)=-(h-x) a(x)$ for solutions $a(x)$ of the Riccati equation.

Lemma 31. Let $K, \kappa \in \mathbb{R}$. Let $y: I \rightarrow \mathbb{R}$ be a solution to the Riccati equation

$$
y^{\prime}+y^{2}+K=0, \quad y(0)=-\kappa,
$$

with maximal interval $I$. Let $h \in I$ and define the function $f: I \rightarrow \mathbb{R}$ by $f(x)=$ $-(h-x) y(x)$. The following inequalities hold:

\begin{tabular}{lcl} 
Condition on $K$ & Condition on $\kappa$ & Inequality \\
\hline$K \geq 0$ & $\kappa \in \mathbb{R}$ & $\min \{0, h \kappa\} \leq f(x) \leq 1$ \\
$K<0$ & $\kappa \geq \sqrt{|K|}$ & $0 \leq f(x) \leq h \kappa$
\end{tabular}

5.2. Totally geodesic boundary. This corresponds to the parts (1) and (2) of Theorem 7 .

Proposition 32. Let $M$ be a compact manifold with nonempty totally geodesic boundary $\Sigma$. Assume that $|K|<\lambda^{2}$, where $\lambda>0$, on $M_{\bar{h}}$. Then, (3) and (4) hold with

$$
\mathbf{A}=\frac{n}{\tilde{h}}+\lambda \quad \text { and } \quad \mathbf{B}=\frac{1}{2}\left(\frac{1}{\bar{h}}+n \lambda\right)
$$

where $\tilde{h}=\min \left\{\frac{\pi}{2 \lambda}, \bar{h}\right\}$.

Moreover, if $-\lambda^{2}<K<0$, then (3) and (4) hold with

$$
\mathbf{A}=\frac{1}{\bar{h}}+\lambda \quad \text { and } \quad \mathbf{B}=\frac{1}{2}\left(\frac{1}{\bar{h}}+n \lambda\right) .
$$

On the other hand, if $0<K<\lambda^{2}$, then (3) and (4) hold with

$$
\mathbf{A}=n \max \left\{\bar{h}^{-1}, \frac{2 \lambda}{\pi}\right\}, \quad \text { and } \quad \mathbf{B}=\frac{1}{2 \bar{h}}
$$


Proof. It follows from Corollary 15 and Lemma 18 that

$$
\tilde{h}=\min \left\{\operatorname{roll}(M), m\left(\lambda^{2}, 0\right)\right\}=\min \left\{\frac{\pi}{2 \lambda}, \bar{h}\right\} .
$$

Moreover, the corresponding comparison functions are

$$
\mu(\delta)=a(\delta)=\lambda \tanh (\lambda \delta) \quad \text { and } \quad b(\delta)=-\lambda \tan (\lambda \delta) .
$$

It follows from Lemma 31 that $-(h-\delta) b(\delta) \leq 1$. Moreover, for every $0<\delta \leq h \leq$ $\tilde{h} \leq \bar{h}$

$$
(h-\delta) \lambda \tanh (\lambda \delta)<\lambda \tilde{h}
$$

Whence,

$$
\overline{\mathbf{A}}(h) \leq(n-1)+\lambda \tilde{h} \quad \text { and } \quad \overline{\mathbf{B}}(h) \leq n \lambda \bar{h} .
$$

One can take

$$
\mathbf{A}=\frac{n}{\tilde{h}}+\lambda \quad \text { and } \quad \mathbf{B}=\frac{1}{2}\left(\frac{1}{\bar{h}}+n \lambda\right) .
$$

In the situation where $K<0$,

$$
\tilde{h}=\min \{\operatorname{roll}(M), m(0,0)\}=\bar{h} .
$$

The comparison functions are

$$
\mu(\delta)=a(\delta)=\lambda \tanh (\lambda \delta) \quad \text { and } \quad b(\delta)=0 .
$$

We have $(h-\delta) a(\delta) \leq \bar{h} \lambda$ and

$$
\overline{\mathbf{A}} \leq \bar{h} \lambda \quad \text { and } \quad \overline{\mathbf{B}} \leq n \bar{h} \lambda .
$$

Thus one can take

$$
\mathbf{A}=\frac{1}{\bar{h}}+\lambda \quad \text { and } \quad \mathbf{B}=\frac{1}{2}\left(\frac{1}{\bar{h}}+n \lambda\right) .
$$

In the case $K>0$ the comparison functions are

$$
\mu(\delta)=a(\delta)=0 \quad b(\delta)=-\lambda \tan (\lambda \delta) .
$$

and as mentioned above $-(h-\delta) b(\delta) \leq 1$. Therefore, one can take

$$
\mathbf{A}=n \max \left\{\bar{h}^{-1}, \frac{2 \lambda}{\pi}\right\}, \quad \text { and } \quad \mathbf{B}=\frac{1}{2 \bar{h}} .
$$

Part (2) of Theorem 7 follows immediately from the proof of Proposition 32

5.3. Horoconvex boundary. This corresponds to the part (3) of Theorem 7 . Our hypothesis on the principal curvature implies local convexity as in [29], but the boundary of $M$ is not necessarily connected.

Proposition 33. Let $M$ be a compact manifold with nonempty boundary $\Sigma$. Assume that $-\lambda^{2} \leq K \leq 0$ on $M_{\bar{h}}$ (where $\lambda>0$ ) and assume that each of the principal curvatures of $\Sigma$ satisfies $\lambda<\kappa_{i} \leq \kappa_{+}$. Then, one can take

$$
\mathbf{A}=n \max \left\{\kappa_{+}, \frac{1}{\bar{h}}\right\} \quad \text { and } \quad \mathbf{B}=\frac{1}{2 \bar{h}} .
$$


Proof. It follows from Corollary 15 and Lemma 18 that

$$
\tilde{h}=\min \left\{\operatorname{roll}(M), m\left(0, \kappa_{+}\right)\right\}=\min \left\{1 / \kappa_{+}, \bar{h}\right\} .
$$

Moreover, the corresponding comparison functions satisfy

$$
\mu(\delta)=a(\delta)=-\lambda \quad \text { and } \quad-b(\delta)=\frac{1}{-\delta+1 / \kappa_{+}} .
$$

It follows that $(\tilde{h}-\delta) a(\delta) \leq 0$. Moreover,

$$
-(h-\delta) b(\delta) \leq\left(\frac{1}{\kappa_{+}}-\delta\right)\left(\frac{1}{-\delta+1 / \kappa_{+}}\right)=1 .
$$

Whence, $\overline{\mathbf{A}} \leq n-1$ and we can take $\overline{\mathbf{B}}=0$ by Remark 26 . Therefore one can take

$$
\mathbf{A}=\frac{n}{\tilde{h}} \quad \text { and } \quad \mathbf{B}=\frac{1}{2 \bar{h}} .
$$

5.4. Positive sectional curvature. This corresponds to the part (4) of Theorem 7

Proposition 34. Let $M$ be a compact manifold with nonempty boundary $\Sigma$. Let $\bar{h}=\operatorname{roll}(M)$ be its rolling radius. Assume that $0<K<\lambda^{2}$, where $\lambda>0$, on $M_{\bar{h}}$. Also assume that each principal curvatures satisfy $0 \leq \kappa_{i} \leq \kappa_{+}$on $\Sigma$. Then, one can take

$$
\mathbf{A}=n \max \left\{\frac{1}{\bar{h}}, \sqrt{\lambda^{2}+\kappa_{+}^{2}}\right\}, \quad \text { and } \quad \mathbf{B}=\frac{1}{2 \bar{h}} .
$$

Proof. It follows from Corollary 15 and Lemma 18 that

$$
\tilde{h}=\min \left\{\operatorname{roll}(M), m\left(\lambda^{2}, \kappa_{+}\right)\right\}=\min \left\{\frac{1}{\lambda}\left(\frac{\pi}{2}-\arctan \left(\frac{\kappa_{+}}{\lambda}\right)\right), \bar{h}\right\} .
$$

Let us show that

$$
\frac{\lambda}{\frac{\pi}{2}-\arctan \left(\frac{\kappa_{+}}{\lambda}\right)} \leq \sqrt{\lambda^{2}+\kappa_{+}^{2}}
$$

Indeed this is equivalent to

$$
\frac{\lambda}{\sqrt{\lambda^{2}+\kappa_{+}^{2}}} \leq \frac{\pi}{2}-\arctan \left(\frac{\kappa_{+}}{\lambda}\right)
$$

Using that $\sin$ is increasing on $[0, \pi / 2]$ this leads to the equivalent

$$
\sin \left(\frac{\lambda}{\sqrt{\lambda^{2}+\kappa_{+}^{2}}}\right) \leq \sin \left(\frac{\pi}{2}-\arctan \left(\frac{\kappa_{+}}{\lambda}\right)\right)=\cos \left(\arctan \left(\frac{\kappa_{+}}{\lambda}\right)=\frac{\lambda}{\sqrt{\lambda^{2}+\kappa_{+}^{2}}},\right.
$$

which is true.

Moreover, the corresponding comparison functions satisfy

$$
\mu(\delta)=a(\delta)=0 \text { and }-b(\delta)=\lambda \tan \left(\lambda \delta+\frac{1}{\lambda} \arctan \left(\frac{\kappa_{+}}{\lambda}\right)\right)
$$


It follows from Lemma 31 that $-(h-\delta) b(\delta) \leq \kappa_{+} h \leq 1$. Whence, $\overline{\mathbf{A}} \leq n-1$, $\overline{\mathbf{B}}=0$, and one can take

$$
\mathbf{A}=n \max \left\{\frac{1}{\bar{h}}, \sqrt{\lambda^{2}+\kappa_{+}^{2}}\right\} \quad \text { and } \quad \mathbf{B}=\frac{1}{2 \bar{h}} .
$$

In some situations, the work of Donnelly and Lee [7] can be used to compute $\bar{h}$ explicitly.

Corollary 35. Let $M$ be a domain in a complete Riemannian manifold. Suppose that $\Sigma$ is connected and convex $\left(\kappa_{-}>0\right)$. Let $\lambda, \kappa_{+}>0$. Then

(1) Suppose that $\Sigma$ is totally geodesic. If $0<K<\lambda^{2}$, then (3) and (4) hold with

$$
\mathbf{A}=\frac{2 n \lambda}{\pi} \quad \text { and } \quad \mathbf{B}=\frac{\lambda}{\pi} .
$$

(2) If $-\lambda^{2} \leq K \leq 0$ and each principal curvature satisfies $\lambda<\kappa_{i}<\kappa_{+}$, then (3) and (4) hold with

$$
\mathbf{A}=n \kappa_{+} \quad \text { and } \quad \mathbf{B}=\frac{1}{2} \kappa_{+} .
$$

(3) If $0<K<\lambda^{2}$ and each principal curvature satisfies $0<\kappa_{i}<\kappa_{+}$, then (3) and (4) hold with

$$
\mathbf{A}=n \sqrt{\lambda^{2}+\kappa_{+}^{2}} \quad \text { and } \quad \mathbf{B}=\frac{1}{2} \sqrt{\lambda^{2}+\kappa_{+}^{2}} .
$$

Proof. The estimates on $\bar{h}$ given in [7, Theorem 3.11, Theorem 3.22] are summarised in the following table.

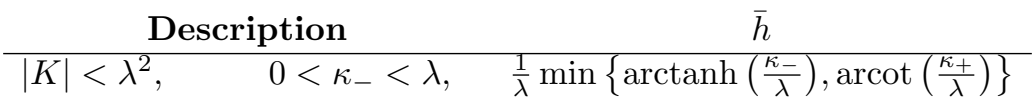

$$
\begin{aligned}
& |K|<\lambda^{2}, \quad \kappa_{-} \geq \lambda, \quad \frac{\operatorname{arcot}\left(\frac{\kappa_{+}}{\lambda}\right)}{\lambda} \\
& -\lambda^{2} \leq K \leq 0, \quad 0<\kappa_{-}<\lambda, \quad \min \left\{\frac{1}{\kappa_{+}}, \frac{\operatorname{arcot}\left(\frac{\kappa_{+}}{\lambda}\right)}{\lambda}\right\} \\
& -\lambda^{2} \leq K \leq 0, \quad \kappa_{-} \geq \lambda \\
& 0<K<\lambda^{2}, \quad \kappa_{+}>\kappa_{-} \geq 0 \quad \frac{\operatorname{arcot}\left(\frac{\kappa_{+}}{\lambda}\right)}{\lambda} \\
& 0<K<\lambda^{2}, \quad \kappa_{-}=\kappa_{+}=0, \quad \frac{\pi}{2 \lambda}
\end{aligned}
$$

In each cases listed in the corollary, it is now enough to replace the value of $\bar{h}$ in Theorem 7 with the value given in the table above. To get part (3), we also use the following inequality which is proved in the proof of Proposition 34.

$$
\frac{\lambda}{\operatorname{arcot}\left(\frac{\kappa_{+}}{\lambda}\right)}=\frac{\lambda}{\frac{\pi}{2}-\arctan \left(\frac{\kappa_{+}}{\lambda}\right)} \leq \sqrt{\lambda^{2}+\kappa_{+}^{2}} .
$$


5.5. Flat manifolds. The goal is to prove Theorem 9 .

Proof. Case 1: It follows from Corollary 15 and Lemma 18 that

$$
\tilde{h}=\min \left\{\operatorname{roll}(M), m\left(0, \kappa_{+}\right)\right\}=\min \left\{1 / \kappa_{+}, \bar{h}\right\} .
$$

Moreover, the corresponding comparison functions are

$$
\mu(\delta)=a(\delta)=\frac{1}{\delta-1 / \kappa_{-}}, \text {and } \quad b(\delta)=\frac{1}{\delta-1 / \kappa_{+}} .
$$

Whence, for every $0<\delta<h$

$$
(h-\delta) a(\delta)=(h-\delta) \mu(\delta) \leq h\left|\kappa_{-}\right|, \quad \text { and } \quad-(h-\delta) b(\delta) \leq 1 .
$$

Thus, $\overline{\mathbf{A}} \leq n-1+h\left|\kappa_{-}\right|$, and $\overline{\mathbf{B}}=h\left|\kappa_{-}\right|$. Therefore, one can take

$$
\mathbf{A}=n \max \left\{\bar{h}^{-1} \kappa_{+}\right\}+\left|\kappa_{-}\right|, \quad \text { and } \quad \mathbf{B}=\frac{1}{2}\left(\bar{h}^{-1}+n\left|\kappa_{-}\right|\right) .
$$

Case 2: $0 \leq \kappa_{i}<\kappa_{+}$. It follows from Corollary 15 and Lemma 18 that

$$
\tilde{h}=\min \left\{\operatorname{roll}(M), m\left(0, \kappa_{+}\right)\right\}=\min \left\{1 / \kappa_{+}, \bar{h}\right\} .
$$

Moreover, the corresponding comparison functions are

$$
\mu(\delta)=a(\delta)=0, \text { and } \quad b(\delta)=\frac{1}{\delta-1 / \kappa_{+}} .
$$

Whence,

$$
-(h-\delta) b(\delta) \leq 1 .
$$

Thus, $\overline{\mathbf{A}} \leq n-1$, and $\overline{\mathbf{B}}=0$. Therefore, one can take

$$
\mathbf{A}=\frac{n}{\tilde{h}}=n \max \left\{\bar{h}^{-1}, \kappa_{+}\right\}, \quad \text { and } \quad \mathbf{B}=\frac{1}{2 \bar{h}} .
$$

Case 3: $\kappa_{-}<\kappa \leq 0$. It follows from Corollary 15 and Lemma 18 that

$$
\tilde{h}=\min \left\{\operatorname{roll}(M), m\left(0, \kappa_{-}\right)\right\}=\bar{h} .
$$

Moreover, the corresponding comparison functions are

$$
\mu(\delta)=a(\delta)=\frac{1}{\delta-1 / \kappa_{-}}, \quad \text { and } \quad b(\delta)=0 .
$$

It follows that $(h-\delta) \frac{1}{\delta-1 / \kappa_{-}} \leq h\left|\kappa_{-}\right|$for $0<\delta<h$, so that one can take

$$
\mathbf{A}=\bar{h}^{-1}+\left|\kappa_{-}\right| \quad \text { and } \quad \mathbf{B}=\frac{1}{2}\left(\bar{h}^{-1}+n\left|\kappa_{-}\right|\right) .
$$

Remark 36. In all estimates above for $\mathbf{B}$, one can consider $\kappa_{-}$as a lower bound for the mean curvature of $\Sigma$ and $\alpha$ as a lower bound on the Ricci curvature. The same estimates remain true. See also Theorem 4. 
5.6. Cylindrical boundaries. Let assume that a neighbourhood of the boundary $\Sigma$ is isometric to $\Sigma \times[0, L)$. In the Riccati equation (15) only the sectional curvature along the normal geodesic appears, and it vanishes in the present example. Because moreover the boundary $\Sigma$ is totally geodesic, we have $\overline{\mathbf{B}}=\overline{\mathbf{A}}=0$ and $\operatorname{roll}(M) \geq L$. Therefore, (3) and (4) hold with $2 \mathbf{B}=\mathbf{A}=\frac{1}{L}$. In particular $\left|\sigma_{j}-\sqrt{\lambda_{j}}\right|<1 / L$. In fact, using the min-max characterization of eigenvalues and separation of variables, it is possible to show that for each $j$ which is larger than the number of connected components of $\Sigma$, one has

$$
\sqrt{\lambda_{j}} \tanh \left(\sqrt{\lambda_{j}} L\right) \leq \sigma_{j} \leq \sqrt{\lambda_{j}} \operatorname{coth}\left(\sqrt{\lambda_{j}} L\right) .
$$

This implies that $\sigma_{j}-\sqrt{\lambda_{j}}$ tends to zero very fast with $\lambda_{j} \rightarrow \infty$.

\section{EXAMPLES AND REMARKS}

In this section, we discuss the necessity of the hypothesis of Theorem 3 and give different kinds of examples to illustrate this.

Example 37. The condition on the rolling radius is clearly a necessary condition. An easy example, with two boundary components, is given as follow: Take $M=$ $T^{n} \times[0, L]$ where $T^{n}$ is a $n$-dimensional flat torus. The sectional curvature of $M$ is 0 , the principal curvatures of $\partial M=T^{n}$ are 0 . As $L \rightarrow 0$, the rolling radius tends to 0 , and $\sigma_{k} \rightarrow 0$ for all $k$ ([1]). However $\lambda_{3}(\partial M)$ is fixed and strictly positive. This contradicts Inequality (3), and shows that we need a lower bound on the rolling radius.

We can construct an example with one boundary component in the spirit of [4. Example 2]. The length of the boundary is bounded, and this insures that $\lambda_{2}$ is bounded away from 0 . The curvature of the boundary is bounded, but as the boundary becomes "close to itself", this allow to construct small $\sigma_{2}$.

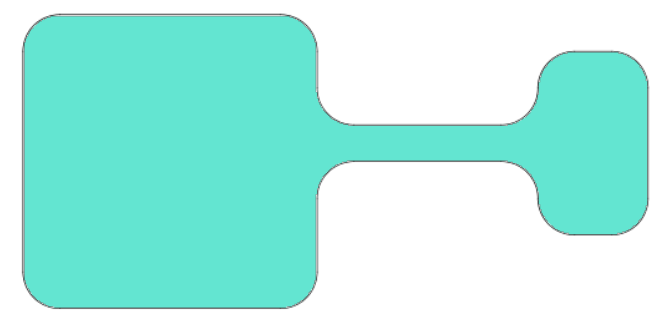

Example 38. The condition on the curvature of the ambient space is also a necessary condition: in Theorem 3.5 of [2], we have constructed an example where the boundary is fixed (which implies that all $\lambda_{i}$ are fixed), its principal curvatures equal to 0 and the rolling radius uniformly bounded from below. However, $\sigma_{2}$ becomes arbitrarily large. This contradicts Inequality (4) and this comes form the fact that the curvature of the ambient space goes to $\infty$.

We can also adapt this example in order to show that without constraint on the curvature, $\sigma_{3}$ may be small and not $\lambda_{3}$, even with a control of the rolling radius. We 
take $\Sigma$ of dimension $n \geq 3$, and consider a Riemannian metric $g_{\varepsilon}=h_{\varepsilon}^{2}(t) g_{0}+d t^{2}$ which coincides with $g$ in a $\epsilon$-neighbourhood of $\Sigma$ in $M$ and such that $h_{\varepsilon}^{2}(t)$ is $\leq \epsilon$ outside a neighbourhood of radius $2 \epsilon$ of $\Sigma$. A direct and simple calculation shows that if $f$ is a function of norm 1 on $\Sigma$, then the Rayleigh quotient of $f$ viewed as a function on $M$ (that is $F(p, t)=f(p)$ ) has a Rayleigh quotient converging to 0 with $\epsilon$. All the Steklov spectrum of $\left(M, g_{\varepsilon}\right)$ goes to 0 with $\epsilon$.

Example 39. One can use Example 6.3 given in the proof of theorem 7 in [6] in order to construct an example with $\sigma_{2}$ bounded from below by a positive constant, but $\lambda_{2}$ arbitrarily small. Example 6.3 consist in gluing together a finite number of fundamental pieces, so that we get a family of example with uniformly bounded geometry (curvature, curvature of the boundary, rolling radius). Moreover, we have shown that $\sigma_{2}$ was uniformly bounded below. At the same time, the boundary is a curve of length going to $\infty$, so that $\lambda_{2}$ goes to 0 .

\section{Appendix: the Riccati equation in Dimension one}

Let $K, \kappa \in \mathbb{R}$. Let $\mathbb{M}_{K}$ be the space-form of constant sectional curvature $K$. Let $\Omega \subset \mathbb{M}_{K}$ be an open connected set with connected smooth umbilical boundary $\Sigma=\partial \Omega \subset \mathbb{M}_{\alpha}$ with principal curvature $\kappa$. We recall that, in this situation, we have to investigate equation (18):

$$
\left\{\begin{array}{l}
y^{\prime}+y^{2}+K=0 \\
y(0)=-\kappa
\end{array}\right.
$$

Our goal is to compute the solution $a(s)$ explicitly and to determine the maximal $m>0$ such that the solution $y$ exists on $[0, m)$ in order to prove Lemma 18 and 19 .

Proof of Lemma 18. In the particular situation where $\kappa^{2}+K=0$, the solution is the constant function $y: \mathbb{R} \rightarrow \mathbb{R}$ defined by $y(t)=-\kappa$. In all other situations, the solution satisfies

$$
-\frac{y^{\prime}}{y^{2}+K}=1 \quad \text { on } I
$$

Integration from 0 to $x \in I$ leads to

$$
-\int_{0}^{x} \frac{y^{\prime}(t)}{y^{2}(t)+K} d t=x
$$

The explicit representation of this integral by elementary functions depend on the sign of the curvature $K$.

Case $K=0$. It follows from

$$
x=-\int_{0}^{x} \frac{y^{\prime}(t)}{y^{2}(t)} d t=\frac{1}{y(x)}-\frac{1}{y(0)}=\frac{1}{y(x)}+\frac{1}{\kappa}
$$

that

$$
y(x)=\frac{1}{x-\frac{1}{\kappa}}=\frac{\kappa}{\kappa x-1}
$$


The maximal existence time therefore is

$$
m(0, \kappa)= \begin{cases}\frac{1}{\kappa} & \text { if } \kappa>0 \\ \infty & \text { if } \kappa \leq 0\end{cases}
$$

Case $K>0$. Let $\lambda>0$ be such that $K=\lambda^{2}$, and observe that

$$
\begin{aligned}
-\int_{0}^{x} \frac{y^{\prime}(t)}{y^{2}(t)+K} d t & =-\frac{1}{\lambda} \int_{0}^{x} \frac{\frac{y^{\prime}(t)}{\lambda}}{\left(\frac{y(t)}{\lambda}\right)^{2}+1} d t \\
& =-\frac{1}{\lambda}\left(\arctan \left(\frac{y(x)}{\lambda}\right)-\arctan \left(\frac{y(0)}{\lambda}\right)\right) \\
& =\frac{1}{\lambda}\left(-\arctan \left(\frac{y(x)}{\lambda}\right)-\arctan \left(\frac{\kappa}{\lambda}\right)\right)
\end{aligned}
$$

Together with 39 this implies that

$$
y(x)=-\lambda \tan \left(\lambda x+\arctan \left(\frac{\kappa}{\lambda}\right)\right)
$$

For $x>0$, this solution is well-defined as long as

$$
\lambda x+\arctan \left(\frac{\kappa}{\lambda}\right)<\pi / 2
$$

That is,

$$
m(K, \kappa)=\frac{1}{\lambda}\left(\frac{\pi}{2}-\arctan \left(\frac{\kappa}{\lambda}\right)\right)
$$

Case $K<0$. Let $\lambda>0$ be such that $K=-\lambda^{2}$. The left-hand-side of 39 is

$$
\begin{aligned}
-\int_{0}^{x} \frac{y^{\prime}(t)}{y^{2}(t)+K} d t & =\frac{1}{\lambda} \int_{0}^{x} \frac{\frac{y^{\prime}(t)}{\lambda}}{1-\left(\frac{y(t)}{\lambda}\right)^{2}} d t \\
& =\frac{1}{\lambda} \int_{y(0) / \lambda}^{y(x) / \lambda} \frac{1}{1-u^{2}} d u=\frac{1}{\lambda} \int_{-\kappa / \lambda}^{y(x) / \lambda} \frac{1}{1-u^{2}} d u
\end{aligned}
$$

Now if $-\kappa / \lambda \in(-1,1)$ then

$$
\frac{1}{\lambda} \int_{-\kappa / \lambda}^{y(x) / \lambda} \frac{1}{1-u^{2}} d u=\frac{1}{\lambda}\left(\operatorname{arctanh}\left(\frac{y(x)}{\lambda}\right)-\operatorname{arctanh}\left(-\frac{\kappa}{\lambda}\right)\right) .
$$

On the other hand, if $|\kappa / \lambda|>1$ then

$$
\frac{1}{\lambda} \int_{-\kappa / \lambda}^{y(x) / \lambda} \frac{1}{1-u^{2}} d u=\frac{1}{\lambda}\left(\operatorname{arcoth}\left(\frac{y(x)}{\lambda}\right)-\operatorname{arcoth}\left(-\frac{\kappa}{\lambda}\right)\right) .
$$

It follows that

$$
y(x)= \begin{cases}\lambda \tanh \left(\lambda x-\operatorname{arctanh}\left(\frac{\kappa}{\lambda}\right)\right) & \text { if }|\kappa|<\lambda \\ \lambda \operatorname{coth}\left(\lambda x-\operatorname{arcoth}\left(\frac{\kappa}{\lambda}\right)\right) & \text { if }|\kappa|>\lambda\end{cases}
$$

The maximal time of existence is

$$
m(y, \kappa)= \begin{cases}\frac{1}{\lambda} \operatorname{arcoth}\left(\frac{\kappa}{\lambda}\right) & \text { if } \kappa>\lambda \\ +\infty & \text { if }|\kappa|<\lambda \\ +\infty & \text { if } \kappa<-\lambda\end{cases}
$$


We can now prove Lemma 19 and Lemma 31 which provide bounds on $f(x)=$ $-(h-x) y(x)$.

Proof of Lemma 19. We use the formula for $y(s)$ given in Lemma 18 to verify the statement in different cases. The proof is a straightforward calculation.

Case 1. $K=\lambda^{2}, \lambda>0$. Here we use the fact that

$$
x \cot (x) \leq 1, \quad \forall x \in[0, \pi),
$$

with equality at $x=0$.

Recall from Lemma 18 that for $K=\lambda^{2}$ we have

$$
-y(s)=\lambda \tan \left(\lambda s+\arctan \left(\frac{\kappa}{\lambda}\right)\right)=\lambda \cot \left(\operatorname{arcot}\left(\frac{\kappa}{\lambda}\right)-\lambda s\right)
$$

for any $0 \leq s \leq \frac{1}{\lambda} \operatorname{arcot}\left(\frac{\kappa}{\lambda}\right)$. We used the identity $\arctan (x)+\operatorname{arcot}(x)=\frac{\pi}{2}$. Thus

$$
-(h-s) y(s) \leq\left(\operatorname{arcot}\left(\frac{\kappa}{\lambda}\right)-\lambda s\right) \cot \left(\operatorname{arcot}\left(\frac{\kappa}{\lambda}\right)-\lambda s\right) \leq 1 \text {. }
$$

Case 2. $K=0$. If $\kappa \leq 0$, then $-y(s) \leq 0$ on $I$. If $\kappa>0$, then $-(h-s) y(s)=$ $(h-s) \frac{\kappa}{1-\kappa s} \leq 1$ if and only if $h<\frac{1}{\kappa}$. Hence, it is clear that in both situations the statement of the lemma holds.

Proof of Lemma 31. We consider two cases separately.

Case 1: $K \geq 0$. From Lemma 19, we know that $f(x) \leq 1$ for $K \geq 0$. It remains to show that $f(x) \geq \min \{0, h \kappa\}$.

Case 1(a). $K=0$. If $\kappa=0$, then $y \equiv 0$ and $f \equiv 0$ and there is nothing more to prove. If $\kappa>0$, then $I=(0,1 / \kappa)$ and $f(x)=-(h-x) \frac{1}{x-1 / \kappa}>0$. If $\kappa<0$, then $I=(0, \infty)$ and $f(x)=-(h-x) \frac{1}{x-1 / \kappa}$. It follows from $(h-x) \leq h$ and $\left(x-\frac{1}{\kappa}\right) \geq \frac{1}{|\kappa|}$ that

$$
-(h-x) \frac{1}{x-1 / \kappa} \geq h \kappa \text {. }
$$

Case 1(b). $K>0$.

From Lemma 19 and its proof we know

$$
f(x)=\lambda(h-s) \cot \left(\operatorname{arcot}\left(\frac{\kappa}{\lambda}\right)-\lambda s\right), \quad \text { with } I=\left(0, \frac{1}{\lambda} \operatorname{arcot}\left(\frac{\kappa}{\lambda}\right)\right) .
$$

If $\operatorname{arcot}\left(\frac{\kappa}{\lambda}\right)-\lambda s \leq \frac{\pi}{2}$, then $f(x) \geq 0$. If $\operatorname{arcot}\left(\frac{\kappa}{\lambda}\right)-\lambda s \geq \frac{\pi}{2}$, then $\cot \left(\operatorname{arcot}\left(\frac{\kappa}{\lambda}\right)-\lambda s\right) \leq$ 0 and

$$
f^{\prime}(x)=-\lambda \cot \left(\operatorname{arcot}\left(\frac{\kappa}{\lambda}\right)-\lambda s\right)+\frac{\lambda^{2}(h-s)}{\sin ^{2}\left(\operatorname{arcot}\left(\frac{\kappa}{\lambda}\right)-\lambda s\right)} \geq 0 .
$$

Therefore, $f(x) \geq f(0)=h \kappa$.

Case 2: $K<0$ and $\kappa \geq \lambda:=\sqrt{|K|}$. 
The maximal existence interval is $I=\left(0, \frac{1}{\lambda} \operatorname{arcoth}(\kappa / \lambda)\right)$ and

$$
f(x)=-(h-x) \lambda \operatorname{coth}(\lambda x-\operatorname{arcoth}(\kappa / \lambda)) .
$$

For any $\gamma \geq 0$ the function $\psi_{\gamma}: \mathbb{R}_{+} \rightarrow \mathbb{R}$ defined by

$$
\psi_{\gamma}(t)=t \operatorname{coth}(t+\gamma)
$$

is increasing. Let $\phi=\frac{1}{\lambda} \operatorname{arcoth}(\kappa / \lambda)$. Observe that for $\gamma=\lambda \phi-\lambda h$

$$
0 \leq f(x)=\psi_{\gamma}(\lambda h-\lambda x) .
$$

It follows that

$$
\begin{aligned}
0 \leq f(x) & =(\lambda h-\lambda x) \operatorname{coth}(\lambda \phi-\lambda x) \\
& =(\lambda h-\lambda x) \operatorname{coth}(\lambda h-\lambda x+\lambda \phi-\lambda h) \leq \lambda h \operatorname{coth}(\lambda \phi)=h \kappa .
\end{aligned}
$$

\section{ACKNOWLEDGMENT}

Part of this work was done while BC was visiting Université Laval. He thanks the personnel from the Département de mathématiques et de statistique for providing good working conditions. Part of this work was done while AG was visiting Neuchâtel. The support of the Institut de Mathématiques de Neuchâtel is warmly acknowledged. AH would like to thank the Mittag-Leffler Institute for the support and for an excellent working condition during the starting phase of the project.

\section{REFERENCES}

[1] B. Colbois, A. El Soufi, and A. Girouard. Isoperimetric control of the Steklov spectrum. J. Funct. Anal., 261(5):1384-1399, 2011.

[2] B. Colbois, A. El Soufi, and A. Girouard. Compact manifolds with fixed boundary and large Steklov eigenvalues. Proc. Amer. Math. Soc., to appear.

[3] B. Colbois, A. Girouard, and K. Gittins. Steklov eigenvalues of submanifolds with prescribed boundary in Euclidean space. The Journal of Geometric Analysis, to appear.

[4] B. Colbois, A. Girouard, and M. Iversen. Uniform stability of the Dirichlet spectrum for rough outer perturbations. J. Spectr. Theory, 3(4):575-599, 2013.

[5] B. Colbois, A. Girouard, and A. Métras. Submanifolds of Euclidean space with prescribed boundary and small Steklov eigenvalues. In preparation.

[6] B. Colbois, A. Girouard, and B. Raveendran. The Steklov spectrum and coarse discretizations of manifolds with boundary. arXiv:1612.07665, 2016.

[7] H. Donnelly and J. Lee. Domains in Riemannian manifolds and inverse spectral geometry. Pacific J. Math., 150(1):43-77, 1991.

[8] J.-H. Eschenburg. Comparison theorems and hypersurfaces. Manuscripta Math., 59(3):295$323,1987$.

[9] J.-H. Eschenburg and E. Heintze. Comparison theory for Riccati equations. Manuscripta Math., 68(2):209-214, 1990.

[10] K. Funano. Eigenvalues of Laplacian and multi-way isoperimetric constants on weighted Riemannian manifolds, 2013. arXiv:1307.3919.

[11] A. Girouard, L. Parnovski, I. Polterovich, and D. A. Sher. The Steklov spectrum of surfaces: asymptotics and invariants. Math. Proc. Cambridge Philos. Soc., 157(3):379-389, 2014.

[12] A. Girouard and I. Polterovich. Spectral geometry of the Steklov problem. J. Spectr. Theory, $7(2): 321-359,2017$.

[13] A. Gray. Tubes, volume 221 of Progress in Mathematics. Birkhäuser Verlag, Basel, second edition, 2004. 
[14] A. Hassannezhad and L. Miclo. Higher order Cheeger inequalities for Steklov eigenvalues. Ann. Sci. Éc. Norm. Supér., to appear.

[15] A. Hassannezhad and A. Siffert. A note on Kuttler-Sigillito's inequalities, 2017. arXiv:1709.09841.

[16] L. Hörmander. The analysis of linear partial differential operators. IV, volume 275 of Grundlehren der Mathematischen Wissenschaften. Springer-Verlag, Berlin, 1985. Fourier integral operators.

[17] P. Jammes. Une inégalité de Cheeger pour le spectre de Steklov. Ann. Inst. Fourier (Grenoble), 65(3):1381-1385, 2015.

[18] H. Karcher. Riemannian comparison constructions. In Global differential geometry, volume 27 of MAA Stud. Math., pages 170-222. Math. Assoc. America, Washington, DC, 1989.

[19] M. Karpukhin. Bounds between Laplace and Steklov eigenvalues on nonnegatively curved manifolds. Electron. Res. Announc. Math. Sci., 24:100-109, 2017.

[20] N. Kuznetsov, T. Kulczycki, M. Kwaśnicki, A. Nazarov, S. Poborchi, I. Polterovich, and B. Siudeja. The legacy of Vladimir Andreevich Steklov. Notices Amer. Math. Soc., 61(1):922,2014

[21] J. M. Lee and G. Uhlmann. Determining anisotropic real-analytic conductivities by boundary measurements. Comm. Pure Appl. Math., 42(8):1097-1112, 1989.

[22] L. Miclo. On hyperboundedness and spectrum of Markov operators. Invent. Math., 200(1):311-343, 2015.

[23] S. Pigola and G. Veronelli. The smooth Riemannian extension problem. arXiv:1606.08320.

[24] I. Polterovich and D. A. Sher. Heat invariants of the Steklov problem. J. Geom. Anal., 25(2):924-950, 2015.

[25] L. Provenzano and J. Stubbe. Weyl-type bounds for Steklov eigenvalues. J. Spectr. Theory, to appear.

[26] M. A. Shubin. Pseudodifferential operators and spectral theory. Springer-Verlag, Berlin, second edition, 2001. Translated from the 1978 Russian original by Stig I. Andersson.

[27] M. E. Taylor. Partial differential equations. II, volume 116 of Applied Mathematical Sciences. Springer-Verlag, New York, 1996.

[28] Q. Wang and C. Xia. Sharp bounds for the first non-zero Stekloff eigenvalues. J. Funct. Anal., 257(8):2635-2644, 2009.

[29] C. Xiong. Comparison of Steklov eigenvalues on a domain and Laplacian eigenvalues on its boundary in Riemannian manifolds. J. Funct. Anal., 275(12):3245-3258, 2018.

[30] L. Yang and C. Yu. A higher dimensional generalization of Hersch-Payne-Schiffer inequality for Steklov eigenvalues. J. Funct. Anal., 272(10):4122-4130, 2017.

Université de Neuchâtel, Institut de Mathématiques, Rue Emile-Argand 11, CH-2000 NeUChâtel, Switzerland

E-mail address: bruno.colbois@unine.ch

Département de mathématiques et de statistique, Université Laval, Pavillon AlexandreVachon, 1045, av. de la Médecine, Québec QC G1V 0A6, Canada

E-mail address: alexandre.girouard@mat.ulaval.ca

University of Bristol, School of Mathematics, University Walk, Bristol BS8 1TW, UK

E-mail address: asma.hassannezhad@bristol.ac.uk 